\title{
Not all disfluencies are are equal: The effects of disfluent repetitions on language comprehension
}

\author{
Lucy J. MacGregor*,a, Martin Corley ${ }^{\mathrm{b}}$, David I. Donaldson ${ }^{\mathrm{a}}$ \\ ${ }^{a}$ Department of Psychology, University of Stirling \\ ${ }^{b}$ Psychology, PPLS, University of Edinburgh
}

\begin{abstract}
Disfluencies can affect language comprehension, but to date, most studies have focused on disfluent pauses such as er. We investigated whether disfluent repetitions in speech have discernible effects on listeners during language comprehension, and whether repetitions affect the linguistic processing of subsequent words in speech in ways which have been previously observed with ers. We used eventrelated potentials (ERPs) to measure participants' neural responses to disfluent repetitions of words relative to acoustically identical words in fluent contexts, as well as to unpredictable and predictable words that occurred immediately postdisfluency and in fluent utterances. We additionally measured participants' recognition memories for the predictable and unpredictable words. Repetitions elicited an early onsetting relative positivity (100-400ms post-stimulus), clearly demonstrating listeners' sensitivity to the presence of disfluent repetitions. Unpredictable words elicited an N400 effect. Importantly, there was no evidence that this effect, thought to reflect the difficulty of semantically integrating unpredictable compared to predictable words, differed quantitatively between fluent and disfluent utterances. Furthermore there was no evidence that the memorability of words was affected by the presence of a preceding repetition. These findings contrast with previous research which demonstrated an N400 attenuation of, and an increase in memorability for, words that were preceded by an er.
\end{abstract}

\footnotetext{
${ }^{*}$ Corresponding author. Address: Psychological Imaging Laboratory, Department of Psychology, University of Stirling, Stirling, FK9 4LA, UK.

Email address: Lucy.MacGregor@gmail.com (Lucy J. MacGregor)
}

Preprint submitted to Brain \& Language

July 6, 2009 
However, in a later (600-900ms) time window, unpredictable words following a repetition elicited a relative positivity. Reanalysis of previous data confirmed the presence of a similar effect following an er. The effect may reflect difficulties in resuming linguistic processing following any disruption to speech.

Key words: Language Comprehension, Disfluency, Speech, ERPs, Repetitions

\section{Introduction}

Speakers are rarely fully fluent, but produce speech which is peppered with pauses, ums and ers, and prolonged or repeated words. These disfluencies, which typically occur when the speaker is uncertain how to continue, form part of the linguistic input which a listener must interpret. Although a number of recent studies have used different measures to demonstrate that disfluencies directly affect the comprehension process, the focus of the majority of these studies has been on disfluent pauses, particularly er (or $u h$ ).

Ers can speed up response times to subsequent target words in word monitoring (Fox Tree, 2001) or object selection tasks (Brennan and Schober, 2001); further, they can lead listeners to predict the upcoming mention of an item deemed less accessible for the speaker from a constrained set of referents (Arnold, Tanenhaus, Altmann, and Fagnano, 2004; Arnold, Hudson Kam, and Tanenhaus, 2007); and they can also affect the ease of semantic integration of subsequent words (Corley, MacGregor, and Donaldson, 2007). Importantly, ers have been shown to affect not only the process of comprehension but its outcome: In offline studies, Bailey and Ferreira (2003) have demonstrated that ers can affect listeners' interpretations of syntactically ambiguous sentences. Hearing a sentence including an er increases the memorability of the subsequent word (Corley et al., 2007), possibly because of an increase in attention (Collard, Corley, MacGregor, and Donaldson, 2008).

Despite the growing evidence for the effects of ers, few researchers have investigated whether other disfluencies have observable effects on the language comprehension system. The present paper focuses on the disfluent repetition 
of words in an utterance. Disfluent repetitions have been observed to occur in similar situations to disfluent pauses (Beattie and Bradbury, 1979; Howell and Sackin, 2001), at a rate of around 1.5 repetitions per 100 words (Bortfeld, Leon, Bloom, Schober, and Brennan, 2001), with the majority of repetitions comprising function words (2.5 per 100 words: Clark and Wasow, 1998). However, repetitions differ from ers in one very important respect. Ers are clearly distinguishable from the propositional content of an utterance on the basis of their phonology (whether or not they are words: Clark and Fox Tree, 2002). In contrast, a repeated word is recognised as disfluent because of its context.

From the listener's perspective, repetition disfluencies raise two interesting questions. The first concerns the recognition of disfluency: Are there discernible effects on listeners of encountering repeated words which are only disfluent by virtue of their context of occurrence? The second concerns the generality of the reported effects of disfluency: Do repeated words affect the comprehension processes in the same way as ers? Like ers, they introduce time into the speech signal, which is associated with difficulty in aspects of speech production including syntactic planning and lexical retrieval (Blackmer and Mitton, 1991; Clark and Wasow, 1998; Maclay and Osgood, 1959). One possibility is that ers and repetitions are both simple consequences of the speaker's difficulties in planning. For example, Blackmer and Mitton (1991) attribute repetitions at the beginning of phonological phrases to an "autonomous restart capability" within the articulator, according to which existing speech plans are restarted when new material fails to arrive in time. If differing disfluencies reflect similar circumstances in speech, we might expect listeners to treat them equivalently. An alternative view is that some repetitions are used by speakers to establish continuity upon resumption of speaking, in contrast to ers which tend to mark the suspension point (Clark and Wasow, 1998). If listeners are sensitive to such a distinction, we might expect different disfluencies to have different consequences.

To answer these questions, we report an experiment based on previous work by Corley et al. (2007). We use event-related potentials (ERPs) to measure participants' neural responses to disfluent repetitions of words, as well as to the 
words that occur immediately post-disfluency. To establish whether repetition disfluencies have consequences for the outcome of this process, we additionally measure participants' recognition memories for the post-disfluent words. Based on earlier work (Collard et al., 2008; Corley et al., 2007), we anticipate that any attentional changes caused by the disfluent repetitions would enhance memory encoding, ultimately resulting in the post-disfluency being better remembered in a subsequent recognition test.

ERPs are well suited to investigations of speech processing because they can provide a continuous record of brain activity while participants are engaged in comprehension. A number of studies have used ERPs to show observable effects of processing repeated words that do not render the stimuli disfluent. Relative to the first occurrence of a word, repeated words in lists and sentences are commonly associated with an attenuation of the N400 (Besson, Kutas, and Van Petten, 1992; Besson and Kutas, 1993; Ledoux, Traxler, and Swaab, 2007; Okita and Jibu, 1998; Rugg, 1985). The effect is particularly clear if the repeated word is presented immediately after its first occurrence (Nagy and Rugg, 1989). Consistent with the predominant interpretation of the N400 as indicating semantic integration difficulty (e.g., Federmeier, Wlotko, De Ochoa-Dewald, and Kutas, 2007; Hagoort, Hald, Bastiaansen, and Petersson, 2004; Van Berkum, Brown, and Hagoort, 1999; Van Berkum, Brown, Hagoort, and Zwitserlood, 2003; Van Petten and Kutas, 1991; for evidence that the N400 is driven by word associations rather than semantic features, see Rhodes and Donaldson, 2008) these studies suggest that second or later mentions of a word are easier to integrate. Importantly, as would be predicted by models of language comprehension, the presence of a discourse context which renders repeated words unpredictable or unnatural can reverse the N400 attenuation. One example of such a reversal is where the repeated words are definite expressions which co-refer (such as Matt went swimming after Matt had dinner). In these cases there is an increase in the N400 for repeated words (Matt) relative to pronoun controls (he), suggesting that there is greater integration difficulty in cases where pronouns would be predicted (Swaab, Camblin, and Gordon, 2004). Clearly, repetition does affect 
linguistic processing, but studies demonstrate that the effects of repeated words are determined by the context in which they occur. Moreover, to date, we do not know of any studies which have focused on the effects of repeated words in spoken language contexts which render them disfluent.

The studies discussed above all focused on the repeated word itself. In another study of repetitions, Fox Tree (1995) instead investigated the effects of the word which occurs immediately following a disfluent repetition. In a word monitoring task, response times to target words which were preceded by repetitions were no slower than those which were preceded by pauses of equivalent length, and in two experiments, they were faster. One interpretation of these findings is that attention was engaged by the repetitions, leading to faster response times, as has been claimed in the case of er (Fox Tree, 2001). However, a plausible alternative explanation is that pauses in the control condition disrupted processing, resulting in slower response times for this condition relative to the repetition condition. Thus evidence for an effect of repetitions on linguistic processing remains equivocal. Moreover, the task for participants was to monitoring for specific words rather than listen for comprehension, which may have affected the outcome of the experiment. In sum, it is entirely possible that repetition disfluencies affect the processing of the words which follow, and the present study provides evidence to support that possibility.

\subsection{The present study}

The present study is based on an experiment reported in Corley et al. (2007). In the earlier experiment, ERPs were recorded as participants listened to utterances which ended in either predictable or unpredictable target words. Half of the utterances were disfluent by virtue of an er occurring immediately prior to the target word. The results showed that that the presence of an er reduced the amplitude of the N400 effect for unpredictable compared to predictable words. An additional recognition memory test was used to show that target words which had been preceded by er during comprehension were better recognised than those which had not. 
In the current experiment, we focus on repetition disfluencies. The disfluent utterances from the previous experiment were discarded, and novel disfluent utterances were created which contained a repetition immediately preceding the utterance-final target word. Table 1 shows an example stimulus set.

Table 1: Example stimulus set comprising two highly constraining sentence frames, crossed with two utterance-final target words, which were predictable or unpredictable in context. Target words are shown in bold. Half the utterances were disfluent and contained a repetition before the target word, indicated in square brackets.

\begin{tabular}{llll}
\hline Predictable & Everyone's got bad habits and mine is biting my & {$[\mathrm{my}]$} & nails \\
& That drink's too hot; I've just burnt my & {$[\mathrm{my}]$} & tongue \\
Unpredictable & Everyone's got bad habits and mine is biting my & {$[\mathrm{my}]$} & tongue \\
& That drink's too hot; I've just burnt my & {$[\mathrm{my}]$} & nails \\
\hline
\end{tabular}

Given the experimental design, the only factor that rendered repeated tokens disfluent was the preceding context. We were therefore able to compare the ERPs elicited in response to repetition disfluencies with those associated with acoustically identical control words. Since the words were repeated immediately (as in Nagy and Rugg, 1989) but their occurrence was not predictable (as in Swaab et al., 2004), the nature of any effect of repetition under these conditions was one empirical question of considerable interest.

Our second interest concerned the effects of repetition disfluencies on the comprehension of words which occurred later in the utterance. Our predictions were based on the premise that repetitions and er disfluencies have similar distributions in speech (Beattie and Bradbury, 1979; Howell and Sackin, 2001), and thus we expected that the ease with which the post-disfluent target word was integrated would be affected by its predictability (as in Corley et al., 2007). Because disfluency tends to precede less predictable items in speech (Beattie and Butterworth, 1979) we hypothesised that the semantic integration disadvantage for unpredictable items would diminish post-disfluency, resulting in a smaller 
N400 difference between unpredictable and predictable target words following repetitions. Because disfluency affects attention (Collard et al., 2008) we expected target words to be more likely to be recognised if they had been initially encountered post-disfluency (Collard et al., 2008; Corley et al., 2007).

\section{Method}

\subsection{Participants}

Sixteen native British English speakers (7 male; mean age 22; range 1935; all right-handed) who reported no hearing or reading difficulties, and had no known neurological impairment, participated for financial compensation or course credit. Informed consent was obtained in accordance with the University of Stirling Psychology Ethics Committee guidelines.

\subsection{Materials}

The stimuli were 160 highly constrained fluent and disfluent utterances ending in predictable (cloze probability 0.84 , range $0.52-1$ ) or unpredictable (cloze probability 0) target words and were based on those used in Corley et al. (2007). Utterances were constructed in pairs such that each predictable word also served as an unpredictable word for a corresponding utterance. Furthermore, predictable and unpredictable targets completed fluent and disfluent utterances so that across participants each target appeared in every condition. This double counterbalancing ensured that targets were perfectly controlled for grammatical class, duration, frequency, imageability, and concreteness and meant that each participant heard all sentence frames and target words once only. Table 1 shows an example material set.

Stimuli were digitally recorded by a female native English speaker at a natural speaking rate. For each utterance the utterance-final word was replaced by the pseudotarget word pen which meant that there were no acoustic cues to the upcoming word. Any prosodic cues to an upcoming $p$ would have been constant across conditions. Following recording, pseudotargets were excised and replaced 
by target words which had been recorded as utterance-final words in separate carrier sentences.

Disfluent utterances were created from fluent utterances by copying the pretarget word and splicing it into the speech stream, after the original, to form a repetition before the utterance-final target word. Thus each disfluent utterance was identical to a fluent counterpart up to the point of the repeated word. The repetition was typically of a single function word (e.g., the or $a$ ) but sometimes more words were repeated (e.g., to the, for the) when this made it easier to obtain utterances without obvious splicing points. A pause of $200 \mathrm{~ms}$ was inserted between the two tokens of the repetition. The duration chosen was based on the pauses which have been observed during naturally occurring repetitions (Fox Tree, 1995, p. 724) and was shortened or lengthened where this resulted, in the experimenters' opinions, in a better sounding recording. No additional silence other than any which existed in the fluent utterance was inserted after the repeated token.

An additional 80 filler utterances of varying constraint were recorded. Forty were fluent and 40 contained disfluencies of various types (repetitions, ers, silent pauses, and repairs) in various locations.

Before presentation, all stimuli were converted to 16-bit $22050 \mathrm{~Hz}$.wav files, and their amplitudes were normalised so that the acoustic volume was approximately matched across stimuli. Four versions of the experiment were created, for counterbalancing purposes, each containing 160 experimental utterances (40 each of fluent predictable, fluent unpredictable, disfluent predictable, and disfluent unpredictable) together with the 80 filler utterances.

\subsection{Procedure}

There were two parts to the experiment. In the first part, participants were told that they would hear a series of utterances which were re-recorded excerpts from natural conversations. Participants were further advised that because the utterances would be heard out of context, some would make more sense than

others. They were instructed to listen for understanding, just as they would in 
a natural situation. There was no other task. To minimise the introduction of artifacts into the EEG recording, it was emphasised to participants that they should relax, keep as still as possible, and fixate their eyes on a cross in the centre of the screen.

One hundred and sixty experimental utterances were presented auditorily, in a random order, interspersed with fillers. Utterances were presented in two blocks lasting approximately 15 minutes each, separated by a break of a few minutes. The start of each utterance was indicated visually (for $250 \mathrm{~ms}$ ) by a yellow fixation cross on a black screen, which flashed blue once (for 250ms) and returned to yellow as the utterance began. The fixation cross remained on the screen for the duration of the utterance to discourage eye movements. Following each utterance the screen was blanked for $1500 \mathrm{~ms}$.

Following the first part of the experiment, participants took part in a surprise recognition memory test for the utterance final 'old' words. These words had been either contextually predictable or unpredictable, and had been heard in either fluent or disfluent contexts. They were interspersed with 160 frequencymatched 'new' foils, which had not been heard at any point in the first part of the experiment. Targets were presented visually, and participants discriminated between old and new words as accurately as possible by pressing one of two response keys with index fingers (counterbalanced across participants). The start of each presentation was indicated by the appearance of a fixation cross, which was replaced by the target word. After a $750 \mathrm{~ms}$ presentation, the screen was blanked for $1750 \mathrm{~ms}$.

\subsection{ERP recording and pre-processing}

Electrophysiological data was recorded and analysed in the Psychological Imaging Laboratory at the University of Stirling (http://www.erps.stir.ac . uk) using methods which are standard in the cognitive electrophysiology field. During the first part of the experiment, EEG was recorded (Neuroscan 4.2 Acquire software, Neuromedical Supplies, http : //www .neuro.com) from $61 \mathrm{Ag} / \mathrm{AgCl}$ electrodes embedded in an elasticated cap, based on an extended version of the 
international 10-20 system (Jasper, 1958). Data were recorded using a left mastoid reference, and re-referenced offline to the average of left and right mastoid recordings. Electro-oculograms (EOGs) were recorded to monitor for vertical and horizontal eye movements. Electrode impedances were kept below $5 \mathrm{k} \Omega$. The analogue EEG and EOG recordings were amplified (band pass filter $0.01-40 \mathrm{~Hz}$ ), and continuously digitised (16 bit) at a sampling frequency of $200 \mathrm{~Hz}$.

Before off-line averaging, the continuous EEG files for each participant were segmented into $1350 \mathrm{~ms}$ epochs, starting $150 \mathrm{~ms}$ before the critical words, and screened for artifacts. Epochs were excluded when any channel became saturated (exceeding $495 \mu \mathrm{V}$ ), when drift (absolute difference in amplitude between the first and last data point of each individual epoch) was greater than $33.75 \mu \mathrm{V}$, or when amplitude on any channel (excluding VEOG) was greater than $75 \mu \mathrm{V}$. A minimum of 16 artefact-free trials was required from each participant, in each condition, to ensure an acceptable signal-to-noise ratio. The screening process resulted in the loss of $36 \%$ of the trials, with no difference between conditions. The effect of eye-blink artifacts was minimised by estimating and correcting their contribution to the ERP waveforms using a regression procedure which involves calculating an average blink from 32 blinks for each participant, and removing the contribution of the blink from all other channels on a point-by-point basis. Waveforms were baseline corrected by subtracting the mean amplitude over the interval preceding the critical word and smoothed over 5 points so that each sampling point represents the average over the two previous and two subsequent points.

Grand average ERPs were formed time-locked to the critical words, in each condition, for each participant, and then averaged over all participants. ERPs were quantified by measuring the mean amplitude over time windows of interest, for each electrode, in each condition, for each participant. Quantitative differences between conditions were assessed using analyses of variance (ANOVAs). Differences in scalp distributions of effects between conditions were assessed after normalisation for amplitude differences using the Max/Min method (McCarthy and Wood, 1985). All analyses made use of Greenhouse-Geisser corrections 
where appropriate, and are reported using corrected $F$ and $p$ values.

\section{Results}

The results are presented in three sections: First, we consider the effects at the repetition itself, second, we consider the effects at the post-repetition target word, and finally we consider the memory results.

\subsection{Effects at the repetition}

Based on visual inspection of the waveforms, ERPs were quantified by measuring the mean amplitude of the ERP difference between repetition and control words over 100-400ms. Initially, the effects were also analysed over shorter time windows of $50-150 \mathrm{~ms}$ and $150-400 \mathrm{~ms}$, but no differences between these time windows were observed, and the results reported here are from the 100-400ms time window only. The effects were not analysed after $400 \mathrm{~ms}$ because of the potential overlap with the effects time-locked to the utterance-final words, which are analysed separately below.

The repetition effect was assessed by forming grand average ERPs timelocked to the repetitions and comparing them to ERPs formed to (acoustically identical) corresponding control words in fluent utterances. Importantly, in an ANOVA with factors of Predictability [predictable, unpredictable], Repetition [repetition, control], Location [F, FC, C, CP, P], Hemisphere [left, right], and Site [superior: electrode $1 / 2$, medial: electrode $3 / 4$, inferior: electrode $5 / 6$ ], no interactions involving both repetition and predictability reached significance The lack of any interactions show that, as expected, there was no evidence for different effects of repetition which occurred before either predictable or unpredictable words. Therefore, the data for the repeated and control words are presented here collapsed over the predictable/unpredictable conditions. This resulted in two conditions, repetition and control, both of which had mean trial numbers of 26 .

Figure 1 shows the relevant ERPs for midline and grouped left and right hemisphere electrodes. Relative to fluent control words, repeated words show 
a positivity which is broadly distributed over the scalp, but appears larger and longer-lasting over central sites, with a slight right hemisphere bias. The effect onsets around $50 \mathrm{~ms}$, is larger over $100-400 \mathrm{~ms}$ and continues until around $600 \mathrm{~ms}$.
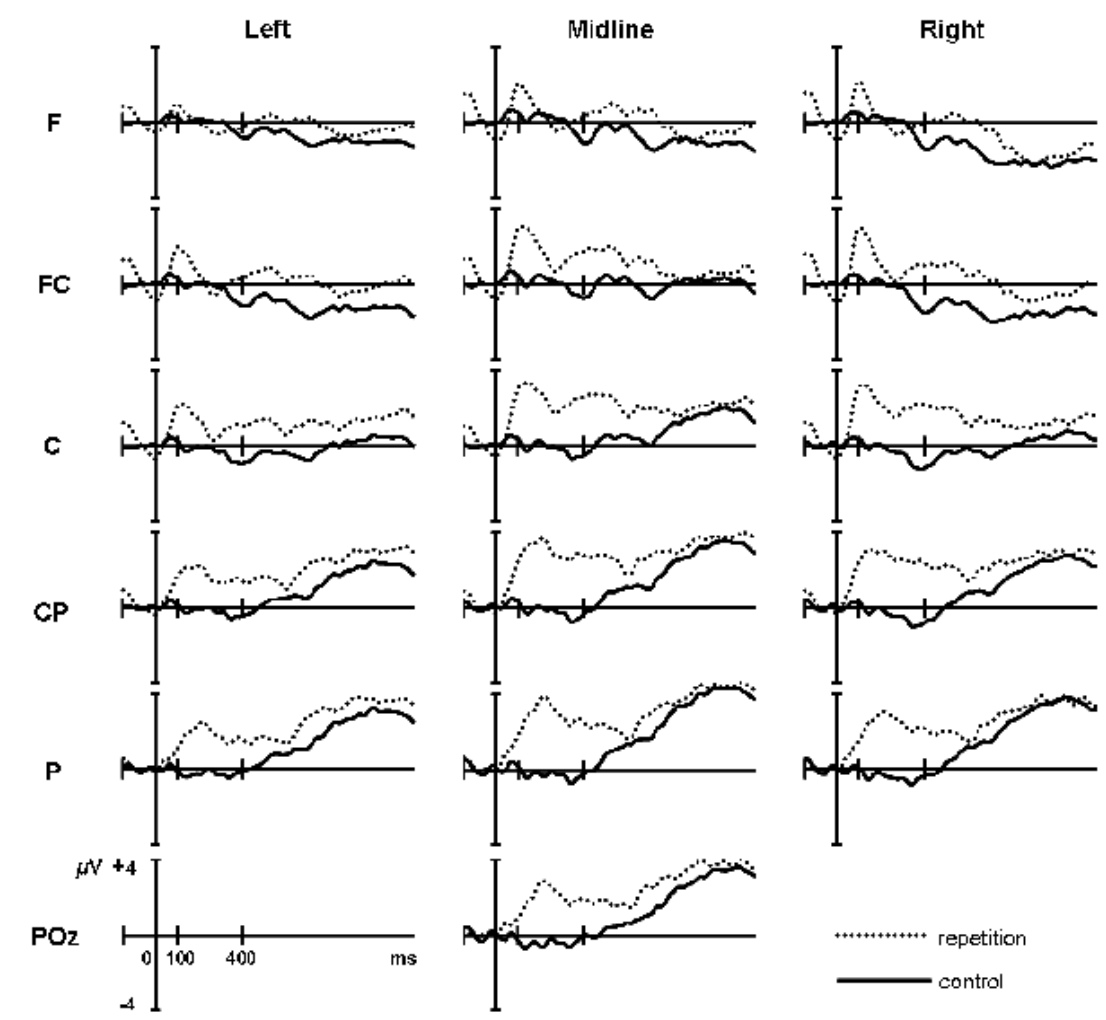

Figure 1: ERPs relative to repeated (dotted lines) or fluent control (dotted lines) word onsets. Positive is plotted up. The central column represents the midline sites (from top: frontal (F), fronto-central (FC), central (C), centro-parietal (CP), parietal (P), occipito-parietal (PO)); the left-hand and right-hand columns represent averages of three electrodes to the left or right of the midline respectively.

The topographic distribution of the repetition effect over 100-400ms is shown in Figure 2.

An ANOVA using the factors of Repetition, Location, Hemisphere, and Site on mean amplitudes over the 100-400ms time window revealed a main effect of repetition $\left[F(1,15)=15.513, \eta_{p}^{2}=.508, p=.001\right]$, reflecting the overall posi- 


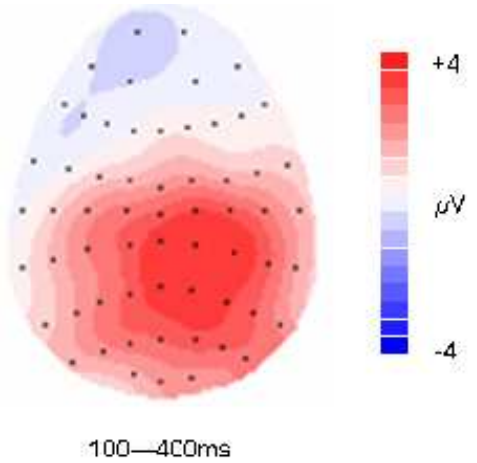

Figure 2: Scalp topography showing the repetition effect over 100-400ms.

tivity for repetitions relative to control words. There were interactions between repetition and location $\left[F(4,60)=24.032, \eta_{p}^{2}=.616, p<.001\right]$, between repetition and hemisphere $\left[F(1,15)=11.109, \eta_{p}^{2}=.425, p=.005\right]$, and between repetition and site $\left[F(2,30)=20.848, \eta_{p}^{2}=.582, p<.001\right]$, reflecting the larger positivity at central/centro-parietal locations, over the right hemisphere, and at superior sites. There was also a three-way interaction between repetition, location and site $\left[F(8,120)=4.690, \eta_{p}^{2}=.238, p=.009\right]$ reflecting a larger positivity over superior sites, at central/centro-parietal locations.

\subsection{Effects at the post-disfluency target}

Effects post-repetition were assessed using grand average ERPs time-locked to the onsets of the utterance-final predictable and unpredictable words. These were created separately for fluent and disfluent utterances, resulting in four conditions: fluent predictable, fluent unpredictable, disfluent predictable, disfluent unpredictable, with mean trial numbers of $26,26,25$, and 26 respectively.

Figure 3 shows relevant ERPs for fluent and disfluent utterances, for midline and grouped left and right hemisphere electrodes. Relative to predictable words, unpredictable words show a negativity over the $300-500 \mathrm{~ms}$ time window which is broadly distributed over the scalp, but appears larger over centroparietal/parietal and midline sites. Although the morphology of the individual 
waveforms is different for fluent and disfluent utterances, similar differences between predictable and unpredictable conditions are evident in each case. The timing and topography of these differences are compatible with their identification as N400 effects (which tend to onset earlier under conditions of auditory presentation: e.g., Connolly and Phillips, 1994; Holcomb and Neville, 1991; Van den Brink, Brown, and Hagoort, 2001).

Following the N400, differences emerge between fluent and disfluent utterances. For fluent utterances, the relative negativity for unpredictable words continues, but appears smaller and more focused at central sites. By contrast, for disfluent utterances, unpredictable words show a relative positivity over frontal and fronto-central sites bilaterally, and over left centro-parietal/parietal sites.

ERPs were quantified over two time windows: the standard N400 time window (300-500ms) and a later (600-900ms) time window based on inspection of the waveforms. Topographic distributions of the effects for fluent and disfluent utterances over the two time windows can be found in Figure 5.

The effect of predictability was assessed first in an omnibus ANOVA with the factors of Fluency [fluent, repetition], Predictability [predictable, unpredictable], Location [F, FC, C, CP, P], Hemisphere [left, right], and Site [superior: electrode 1/2, medial: electrode 3/4, inferior: electrode 5/6]. Subsequent analyses concentrated on the effects for fluent and disfluent conditions separately. When no effects involving hemisphere were found, further analyses concentrated on midline electrodes, using the factors of Predictability [predictable, unpredictable] and Location [Fz, FCz, Cz, CPz, Pz, POz].

To evaluate the difference in the scalp distributions of the predictability effects between fluent and disfluent conditions, the data were additionally normalised and analysed using ANOVAs with the factors of Fluency [fluent, disfluent] and Site [61 electrodes] or Fluency [fluent, disfluent] and Location [Fz, FCz, $\mathrm{Cz}, \mathrm{CPz}, \mathrm{Pz}, \mathrm{POz}$. Differences in scalp distributions of the predictability effects were also evaluated over time for fluent and disfluent conditions separately, using ANOVAs with the factors of Window [early, late] and Site [61 electrodes]. 

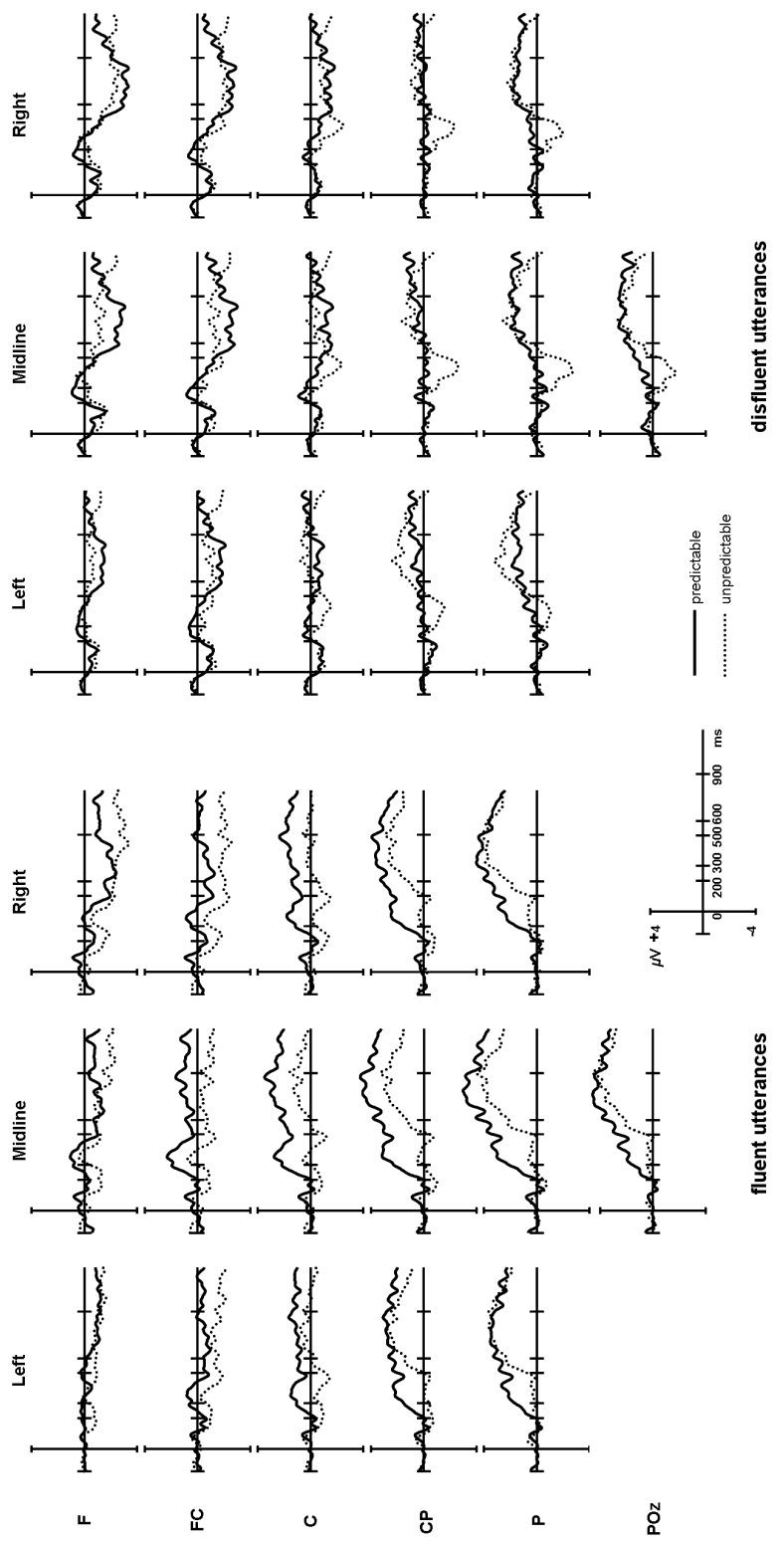

Figure 3: ERPs for fluent (left) and disfluent (right) utterances relative to predictable (solid lines) or unpredictable (dotted lines) target word onsets. Positive is plotted up. The central column of each panel represents the midline sites (from top: frontal (F), fronto-central (FC), central (C), centro-parietal (CP), parietal (P), occipito-parietal (PO)); the left-hand and righthand columns represent averages of three electrodes to the left or right of the midline respectively. 


\subsection{1. $300-500 \mathrm{~ms}$}

Over the 300-500ms time window, an omnibus ANOVA with factors of Fluency, Predictability, Location, Hemisphere and Site showed a a main effect of predictability $\left[F(1,15)=18.16, \eta_{p}^{2}=.557, p=.001\right]$, but no indication of any difference between these effects for fluent and disfluent conditions. Since no effects involving hemisphere were found, a further comparison of the effects for fluent and disfluent conditions was performed at the midline electrodes with the factors of Fluency and Location. Again, there was an expected main effect of predictability $\left[F(1,15)=16.36, \eta_{p}^{2}=.522, p=.001\right]$, reflecting a relative negativity for unpredictable words. There was also a main effect of fluency $\left[F(1,15)=10.15, \eta_{p}^{2}=.404, p=.006\right]$, reflecting the greater overall positivity of the ERPs for fluent utterances. Importantly, there was still no evidence of differences in the effects of predictability between fluent and disfluent conditions.

There was no indication of any differences in the effect of predictability between fluent and disfluent conditions. Because this differed from previous findings using er (Corley et al., 2007), we decided to explore the effects for fluent and disfluent conditions separately, to establish the existence of N400 effects and to compare the topographies of these effects. For fluent utterances there was a main effect of predictability $\left[F(1,15)=17.76, \eta_{p}^{2}=.542, p=.001\right]$ and an interaction between predictability and site $\left[F(2,22)=5.26, \eta_{p}^{2}=.259\right.$, $p=.025]$, reflecting a relative negativity for unpredictable words which was larger towards the midline. For disfluent utterances, there was a main effect of predictability $\left[F(1,15)=6.65, \eta_{p}^{2}=.307, p=.021\right]$ reflecting a relative negativity for unpredictable words, but no other significant effects.

Since no effects involving hemisphere were found for either fluent or disfluent conditions, further analyses focused on effects at the midline electrodes. For fluent utterances, there was a main effect of predictability $[F(1,15)=16.222$, $\left.\eta_{p}^{2}=.520, p=.001\right]$ and an interaction between predictability and location $\left[F(5,75)=4.052, \eta_{p}^{2}=.213, p=.032\right]$, reflecting a relative negativity which was larger at more posterior locations. Disfluent utterances showed a main 
effect of predictability $\left[F(1,15)=5.635, \eta_{p}^{2}=.273, p=.031\right]$, again reflecting a relative negativity.

As a final check that the N400s for fluent and disfluent conditions were equivalent, we performed an ANOVA on the normalised data to assess for topographic differences. There was no evidence of a distributional difference between the effects for fluent and disfluent conditions over the $300-500 \mathrm{~ms}$ time window, neither when data from all electrodes were included $\left[F_{\mathrm{S}}<1\right]$, nor when data from just the midline electrodes were included $\left[F_{\mathrm{s}}<1\right]$. As there is no evidence that the scalp topographies differ between the fluent and disfluent conditions, there is no reason to suppose that different neural generators are responsible for the recorded effects of predictability. ${ }^{1}$

\subsection{2. $600-900 \mathrm{~ms}$}

Over the 600-900ms time window an ANOVA with factors of Fluency, Predictability, Location, Hemisphere, and Site revealed an interaction between fluency and predictability $\left[F(1,15)=6.001, \eta_{p}^{2}=.286, p=.027\right]$, indicating a difference between the predictability effects in fluent and disfluent conditions. There were also interactions between predictability, location and site $\left[F(8,120)=14.001, \eta_{p}^{2}=.483, p<.001\right]$, and a main effect of fluency $[F(1,15)=$ $\left.11.544, \eta_{p}^{2}=.435, p=.004\right]$. A midline ANOVA including factors of Predictability and Fluency also showed an interaction between fluency and predictability $\left[F(1,15)=7.795, \eta_{p}^{2}=.342, p=.014\right]$. The normalised analyses showed no significant effects.

\footnotetext{
${ }^{1}$ We conducted a further set of analyses using a time window enlarged by $40 \%$ over the standard 300-500ms window. An ANOVA using this window (from 260-540ms) including factors of Fluency, Predictability, Hemisphere and Site showed that the results did not differ from those reported above. There was no interaction between fluency and predictability, and other effects were also in line with the previous analysis [main effect of predictability: $F(1,15)=15.468, \eta_{p}^{2}=.508, p=.001$; interaction between predictability and location: $F(4,60)=5.428, \eta_{p}^{2}=.266, p=.027$; interaction between predictability and site: $F(2,30)=$ $\left.4.295, \eta_{p}^{2}=.223, p=.05\right]$. Separate analyses of the fluent and disfluent materials using the extended time window did not differ from those reported above.
} 
Fluent and disfluent conditions were analysed separately, and ANOVAs with the factors of Predictability, Location, Hemisphere, and Site provided no evidence that the distributions of the effects were lateralised in either case. For the fluent condition there were interactions between predictability and site $\left[F(2,30)=16.36, \eta_{p}^{2}=.522, p=.001\right]$, and between predictability, location and site $\left[F(8,120)=7.548, \eta_{p}^{2}=.335, p=.004\right]$, reflecting a larger negativity towards midline sites, and more so at central locations. For disfluent utterances there was an interaction between predictability, location and site $\left[F(8,120)=7.714, \eta_{p}^{2}=.340, p=.002\right]$, reflecting a relative positivity over the frontal location which was larger at superior sites.

\subsubsection{Effects over time}

Finally, we investigated the distributions of effects over time, separately for fluent and disfluent stimuli. ANOVAs performed on normalised data with the factors of Window and Site provided no evidence of distributional differences between the effects over the 300-500ms and 600-900ms time windows for fluent conditions. By contrast, for disfluent conditions there was a significant interaction between Window and Site $\left[F(60,600)=2.952, \eta_{p}^{2}=.164, p=.032\right]$, suggesting a distributional difference between the effects over the two time windows, in particular at midline sites. Specifically, a negative-going effect in the earlier time window develops into a positive-going effect in the later time window.

\subsection{Memory performance}

Memory performance was quantified as the probability of correctly identifying old (previously heard) words. To control for differences in individual memory performance, we treated stimulus identity as a random factor. ${ }^{2}$

\footnotetext{
${ }^{2}$ Traditional adjustments for individual error-rates, such as $d^{\prime}$, are inappropriate here, since the properties of 'old' stimuli are determined by their context of occurrence and hence there are no comparable categories of 'new' stimuli. Using stimulus identity as a random factor ensures that per-participant biases to respond "old" or "new" are controlled for across the
} 
Overall, $61 \%$ of the old words were correctly recognised (false alarm rate $22 \%$ ). Figure 4 shows the recognition probability of utterance-final words by fluency and predictability.
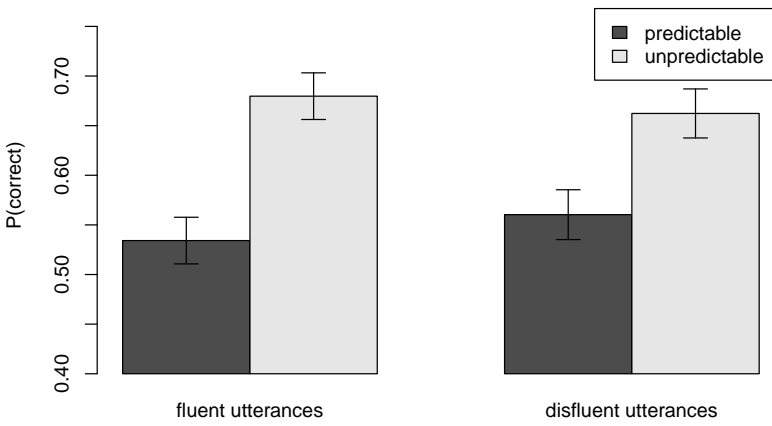

Figure 4: Memory performance for utterance-final words which were originally predictable (black bars) or unpredictable (grey bars) in their contexts, for fluent and disfluent conditions. Error bars represent one standard error of the mean.

An ANOVA carried out with the factors of Fluency [fluent, disfluent] and Predictability [predictable, unpredictable] showed that words that had been unpredictable in their contexts were more likely to be correctly recognised than words that had been predictable $\left[67 \%\right.$ vs. $55 \%: F(1,133)=27.12, \eta_{p}^{2}=.169$, $p<.001]$. This was the case for those which had occurred in fluent [68\% vs. $54 \%$ : $t(133)=4.757, p<.001]$ or in disfluent $[66 \%$ vs. $56 \%: F(133)=3.399, p<.001]$ utterances. There were, however, no effects involving disfluency [interaction

experiment.

Twelve target words were inadvertently repeated in the experiment, resulting in 148 distinct targets. Analysis with data from the repeated targets removed did not affect the outcome. One word (party) was never responded to by participants within the allocated time, resulting in no data for this item. Presentation of one other word (garden) was corrupted. These items were excluded from the analyses. 
between disfluency and predictability: $F(1,133)=1.34, \eta_{p}^{2}=.010, p<.249$; main effect of disfluency: $F<1$ ].

\section{Discussion}

The present experiment investigated whether listeners are affected by the presence of disfluent repetitions encountered during language comprehension, and whether the processing of words which follow a repetition are affected in the same ways that have been observed previously with ers (Corley et al., 2007). Repetitions elicited a relative positivity in the ERP waveform, reliable over a 100-400ms time window, relative to acoustically identical control words in fluent utterances. This positivity provides clear evidence that listeners were sensitive to the disfluencies they encountered. As expected, unpredictable words elicited a relative negativity compared to predictable words, identifiable as a standard N400 effect. In contrast to ers, which have been associated with an attenuation of the N400 to subsequent words, disfluent repetitions preceding the target words, did not lead to a difference in amplitude (or topography) of the effect in the $300-500 \mathrm{~ms}$ time window. Beyond $500 \mathrm{~ms}$, however, the effects elicited by fluent and disfluent utterances differed. During a later (600-900ms) time window, unpredictable words in fluent utterances showed a relative negativity, which appeared to be a continuation of the N400 observed in the earlier window. By contrast, unpredictable words in disfluent utterances gave rise to a positivity over frontal sites. Although this late positivity shows that there is a difference between processing fluent and disfluent utterance, performance on the recognition task was not affected by disfluency, as would be expected based on the lack of differences in the $300-500 \mathrm{~ms}$ time window. Direct comparisons of the effects of particular types of (predictable or unpredictable) target between fluency conditions are not warranted by the present experimental design, because the pre-stimulus ERP baselines are obtained from sentences which include an extra word in the repetition condition. In the present study this extra word is itself associated with a positivity, making the problem particularly salient. 
The memory results appear to pattern with the post-disfluency results in the 300-500ms time window, in that there are no differences attributable to fluency. The equivalent performances in memory across conditions lends credence to the suggestion made by Corley et al. (2007) that their finding of a disfluency advantage in recognition relates to a difference in processing indexed by the N400.

\subsection{Listeners are sensitive to disfluent repetitions}

Hearing a repeated word in a disfluent context resulted in a positive-going shift in the ERP waveform relative to the ERPs formed to acoustically identical control words. This positivity has a similar scalp distribution to the P600, which has previously been associated with syntactic repair or reanalysis (Friederici, 1995, 2002; Friederici, Hahne, and Mecklinger, 1996; Gunter, Stowe, and Mulder, 1997), and more recently with the resolution of conflict between what has been encountered and what would be predicted based on previous experience (Kolk, Chwilla, van Herten, and Oor, 2003; Van Herten, Kolk, and Chwilla, 2005, 2006; Vissers, Chwilla, and Kolk, 2006; see also Kuperberg, 2007, for an alternative conflict-based mechanism). Since repetitions in the present experiment tend to introduce syntactically illegal sequences (e.g., my my in I've just burnt my my tongue), the antecedent conditions are also compatible with a P600 interpretation. ${ }^{3}$

The repetition-related positivity onsets earlier than is typical for the P600 (early P600 onsets are usually around 200ms: Kutas, Van Petten, and Kluender, 2006). In the present case, the early onset of the effect (approximately 50ms) may reflect the ease of detection of a repetition: Since legal repeated-word sequences are extremely rare, listeners may not need to process the structure of a disfluent repetition in much detail in order to decide that it is illegal. Alter-

\footnotetext{
${ }^{3}$ One continuing suggestion is that the P600 is a member of the P300 family (e.g., Coulson, King, and Kutas, 1998; Gunter et al., 1997), but other evidence suggests a distinction (Frisch, Kotz, Von Cramon, and Friederici, 2003; Osterhout and Hagoort, 1999).
} 
natively, the early onset may be attributable to simple detection of a stimulus repetition, which would result in an overlapping but distinctive earlier positive effect such as the P2, an ERP component related to sensory or perceptual processing of stimuli. Although there is no evidence in the current data, the presence of an early positivity is compatible with previous studies that have shown relative positivities to repeated stimuli. For example, an early positivity (30$250 \mathrm{~ms}$ ) with a bilateral fronto-central distribution has been shown in response to repeated tones (Haenschel, Vernon, Dwivedi, Gruzelier, and Baldeweg, 2005) and this has been linked to sensory memory formation.

While it is not possible to determine the exact functional interpretation in a single experiment, it is clear that listeners quickly detect a repetition disfluency. The ERP component associated with this detection occurs early, suggesting that it may be a sensory response to a repeated word. However, the distribution is posterior and the effect is long-lasting, similar to later-occurring P600 effects which index the effects of linguistic or memory-based systems. A speculation would be that listeners are sensitive to both sensory and linguistic properties of repetitions, and that the ERP reflects the interaction between exogenous and endogenous neural generators. The primary consequence of this sensitivity is in the processing of subsequent words, as discussed in section 4.3 below.

\subsection{Disfluent repetitions show different effects to ers on the processing of sub- sequent words}

Repetition disfluencies did not affect the N400 associated with target words or the likelihood of later recognising those words. This contrasts with the case er, where there is clear evidence that the N400, and memory for subsequent words, are affected (Corley et al., 2007). A straightforward interpretation of these findings would suggest that listeners were sensitive to different functions attributed to different types of disfluency, in line with the type of distinction proposed by Clark and Wasow (1998).

Clark and Wasow suggest that repetitions can be used by speakers to serve more than one purpose, and a possible reason that repetition effects are not 
found in the present experiment may lie in a distinction originally proposed by Heike (1981), between repetitions which are followed by silence and those which are not. In line with the majority of observed repetitions (Shriberg, 1995), our materials did not include an additional pause after the repetition. According to Heike, the repeated elements in such cases may mark the resumption of fluent speech following a minor disruption, rather than constituting an interruption in themselves.

From the point of view of the listener, repetitions, even if produced as an automatic consequence of speaker difficulty (e.g., Blackmer and Mitton, 1991), differ from ers in ways which could account for different effects in processing. First, repetitions are lexicalised and are therefore not immediately distinct from the surrounding lexical context, unlike interruptions of debatable lexical status such as ers. An account which focuses on the lexical nature of the disfluency would predict that the effects of other lexical interruptions, for example lexical fillers such as like or y'know, would be similar to those of repetitions. Second, repetitions, unlike pauses, are probably part of the context into which subsequent words are integrated. Although they delay the onset of subsequent new information, conceptualisation of repetitions as a form of 'delay' from the listener's perspective may not be valid, because there is no delay between the repeated word and the subsequent new word. By contrast, ers clearly introduce a delay between the new information and the context into which it must be integrated. An account which attributes the significant effects to the introduction of a delay would predict that the effect of lexical fillers such as like or y'know, would be similar to those of ers.

The potential classification of types of disfluency requires further investigation to determine whether disfluency effects on listeners are lexical or delay oriented, and whether the disfluencies themselves reflect automatic or deliberate speech processes. Indeed it is unclear whether any of these possibilities exclude others. Regardless, it remains clear that repetitions do affect processing, as evidenced by effects on the processing of target words in the later time window. We now turn our attention to the late positivity found for unpredictable words 
when there has been a repetition disfluency.

4.3. Disfluent repetitions show a similar effect to ers on the processing of subsequent words

The timing, distribution, and antecedent conditions of the late positivity are compatible with its identification as a Late Positive Complex (LPC), a positive deflection in the waveform, occurring approximately 500-900 ms after stimulus onset, which has with a frontal focus and may be more prominent over the left hemisphere. Because this positivity depends on predictability, it is unlikely to reflect any ongoing effect of the repetitions, and we therefore attribute it to the target words. Indeed, the LPC has been observed in conditions often associated with the elicitation of an N400. It has been observed in response to unexpected words completing highly constrained sentences (Federmeier et al., 2007), idioms (Moreno, Federmeier, and Kutas, 2002), or stories (Salmon and Pratt, 2002) relative to the most expected words, and to probe words which are unrelated to preceding jokes (Coulson and $\mathrm{Wu}, 2005$ ) relative to semantically related controls. It is attenuated for repeated words in a sentence context relative to their first presentation (Van Petten, Kutas, Kluender, Mitchiner, and McIsaac, 1991).

Functional interpretations of the LPC are typically related to aspects of memory control. This is largely because the frontal distribution of the LPC is similar to positivities that are observed in studies of memory and are associated with retrieval effort (Ranganath and Paller, 1999; Rugg, Allan, and Birch, 2000), or attempts to retrieve source information from memory (Senkfor and Van Petten, 1998). Furthermore, the distribution of the LPC is consistent with a generator in the left inferior prefrontal cortex (Coulson and Wu, 2005), a brain region which is often activated during memory tasks, particularly those involved in semantic processing (Gabrieli, Poldrack, and Desmond, 1998; for a review, see Buckner, 2003).

The presence of the late relative positivity for unpredictable words in the current study is consistent with the memory control account of the LPC. However, two competing explanations are equally plausible. The effect may be associated 
with retrieval of the preceding context and suppression of semantic information associated with the most predictable word (cf. Federmeier et al., 2007), or processes involved with updating working memory (cf. Van Petten et al., 1991). From a theoretical perspective, memory control processes are likely to be engaged as participants attempt to resume structural and, particularly, semantic interpretation of the message after a suspension in interpretation caused by the interruption. Regardless of which of these interpretations is correct, if the presence of the positivity is dependent on the interruption to speech, we would expect a similar effect to be observed following other disfluencies such as ers. We therefore analysed data from Corley et al. (2007) using the same strategy used for the $600-900 \mathrm{~ms}$ analysis of the repetition data in the current experiment. Figure 5 shows the topographic distributions of the effects for fluent and disfluent utterances which included an er over the $300-500 \mathrm{~ms}$ and $600-900 \mathrm{~ms}$ time windows, together with the effects from the present experiment for comparison.

For fluent utterances from Corley et al. (2007), an ANOVA of effects at 600$900 \mathrm{~ms}$ showed a marginal effect of predictability $\left[F(1,11)=4.392, \eta_{p}^{2}=.285\right.$, $p<.060]$, reflecting the fact that the N400 continues, although in a weaker

form, throughout this window. For disfluent utterances, there was a three-way interaction between predictability, location and site $\left[F(8,88)=4.344, \eta_{p}^{2}=.286\right.$, $p=.026]$, reflecting a relative positivity for unpredictable words over the frontal sites close to the midline. As is clear from a comparison of the panels in Figure 5 , this positivity is similar to the effect observed in the present study. Thus unpredictable words elicit a similar late effect following repetitions and ers, compatible with the proposal that the positivity is related to the impact of the disruption.

\section{Conclusions}

It is well known that listeners are sensitive to the presence of disfluencies encountered during comprehension, but the majority of studies to date have focused on the disfluent pause er. The present study focused on repetition 

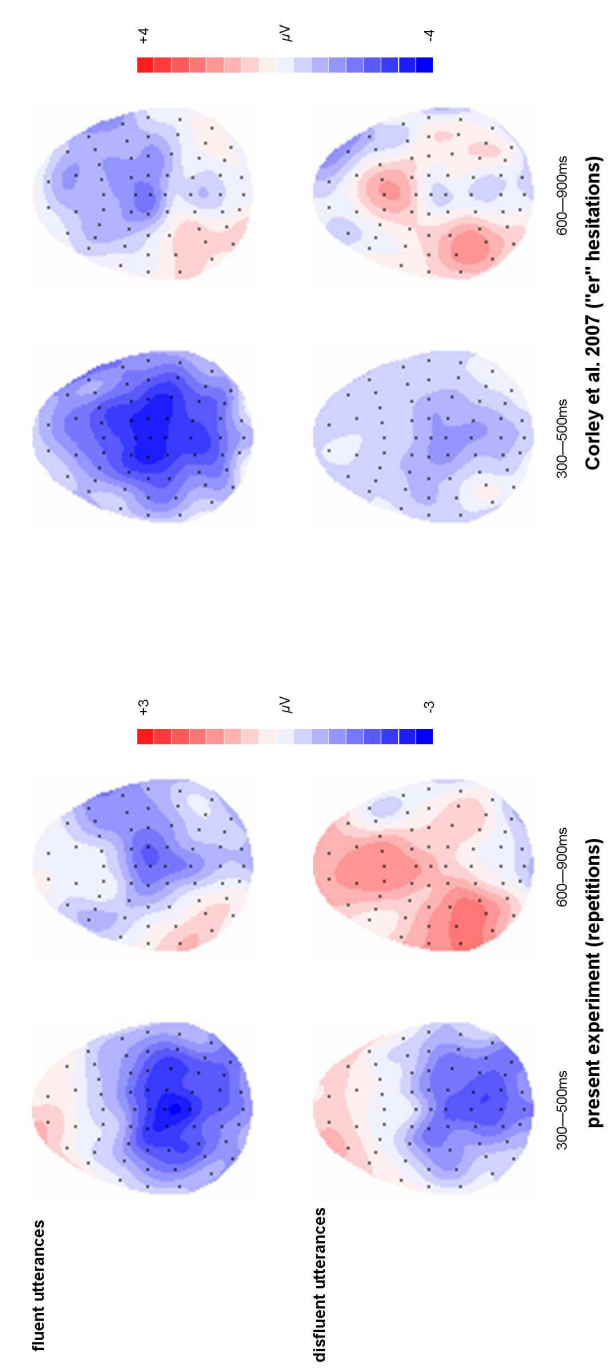

Figure 5: Scalp topographies showing the predictability effects from the present study (left panel) and from Corley et al. (2007) (right panel) over two time windows: 300-500ms and 600-900ms. Data are shown for fluent utterances (top) and for disfluent (bottom) utterances which included either a repetition (left panel) or an er (right panel). 
disfluencies. ERPs revealed that repetitions can be detected within $50 \mathrm{~ms}$ and that following their detection processes of repair and reanalysis are engaged. However, the ERP record provides no evidence that repetitions affect the ease with which subsequent words can be integrated into the discourse as a function of their predictability (as indexed by the magnitude of the N400 effect). This finding stands in stark contrast to the effects of ers observed in previous studies where the N400 effect was attenuated following disfluency, suggesting that, at least from the perspective of the listener, not all disfluencies are equal. Nonetheless, the ERPs revealed an effect of repetitions on the processing of post-disfluent words in a later time window, which may reflect an increase in the difficulty associated with resuming structural and semantic interpretation following an unexpected interruption to an otherwise fluent utterance.

\section{Acknowledgements}

We thank Catriona Bruce for help with data collection and two anonymous reviewers for helpful comments on earlier versions of this paper. The research was partially supported by the Economic and Social Research Council (LJM). DID is a member of the SINAPSE Collaboration (www . sinapse .ac.uk), a Pooling Initiative funded by the Scottish Funding Council and the Chief Scientist Office of the Scottish Executive.

\section{References}

Arnold, J. E., Hudson Kam, C. L., Tanenhaus, M. K., 2007. If you say thee uh you are describing something hard: The on-line attribution of disfluency during reference resolution. Journal of Experimental Psychology: Learning, Memory and Cognition 33, 914-930.

Arnold, J. E., Tanenhaus, M. K., Altmann, R. J., Fagnano, M., 2004. The old and thee, uh, new. disfluency and reference resolution. Psychological Science $15,578-582$. 
Bailey, K. G. D., Ferreira, F., 2003. Disfluencies affect the parsing of gardenpath sentences. Journal of Memory and Language 49, 183-200.

Beattie, G. W., Bradbury, R. J., 1979. An experimental investigation of the modifiability of the temporal structure of spontaneous speech. Journal of Psycholinguistic Research 8, 225-248.

Beattie, G. W., Butterworth, B. L., 1979. Contextual probability and word frequency as determinants of pauses and errors in spontaneous speech. Language and Speech 22, 201-211.

Besson, M., Kutas, M., 1993. The many facets of repetition: A cued-recall and event-related potential analysis of repeating words in the same versus different sentence contexts. Journal of Experimental Psychology: Learning, Memory, and Cognition 19, 1115-1133.

Besson, M., Kutas, M., Van Petten, C., 1992. An event-related potential (ERP) analysis of semantic congruity and repetition effects in sentences. Journal of Cognitive Neuroscience 4, 132-149.

Blackmer, E. R., Mitton, J. L., 1991. Theories of monitoring and the timing of repairs in spontaneous speech. Cognition 39, 173-94.

Bortfeld, H., Leon, S. D., Bloom, J. E., Schober, M. F., Brennan, S. E., 2001. Disfluency rates in conversation: Effects of age, relationship, topic, role, and gender. Language and Speech 44, 123-147.

Brennan, S. E., Schober, M. F., 2001. How listeners compensate for disfluencies in spontaneous speech. Journal of Memory and Language 44, 274-296.

Buckner, R. L., 2003. Functional-anatomic correlates of control processes in memory. Journal of Cognitive Neuroscience 23, 3999-4004.

Clark, H. H., Fox Tree, J. E., 2002. Using uh and um in spontaneous speaking. Cognition 84, 73-111. 
Clark, H. H., Wasow, T., 1998. Repeating words in spontaneous speech. Cognitive Psychology 37, 368-407.

Collard, P., Corley, M., MacGregor, L. J., Donaldson, D. I., 2008. Attention orienting effects of hesitations in speech: Evidence from ERPs. Journal of Experimental Psychology: Learning, Memory and Cognition 34, 696-702.

Connolly, J. F., Phillips, N. A., 1994. Event-related potential components reflect phonological and semantic processing of the terminal word of spoken sentences. Journal of Cognitive Neuroscience 6, 256-266.

Corley, M., MacGregor, L. J., Donaldson, D. I., 2007. It's the way that you, er, say it: Hesitations in speech affect language comprehension. Cognition 105, 658-668.

Coulson, S., King, J. W., Kutas, M., 1998. Expect the unexpected: Eventrelated brain response to morphosyntactic violations. Language and Cognitive Processes 13, 21-58.

Coulson, S., Wu, Y. C., 2005. Right hemisphere activation of joke-related information: An event-related brain potential study. Journal of Cognitive Neuroscience 17, 494-506.

Federmeier, K. D., Wlotko, E. W., De Ochoa-Dewald, E., Kutas, M., 2007. Multiple effects of sentential constraint on word processing. Brain Research $1146,75-84$.

Fox Tree, J. E., 1995. The effects of false starts and repetitions on the processing of subsequent words in spontaneous speech. Journal of Memory and Language 34, 709-738.

Fox Tree, J. E., 2001. Listeners' uses of um and uh in speech comprehension. Memory and Cognition 29, 320-326.

Friederici, A. D., 1995. The time course of syntactic activation during language processing: A model based on neuropsychological and neurophysiological data. Brain and Language 50, 259-281. 
Friederici, A. D., 2002. Towards a neural basis for auditory sentence processing. Trends in Cognitive Science 6, 78-84.

Friederici, A. D., Hahne, A., Mecklinger, A., 1996. Temporal structure of syntactic parsing: Early and late event-related brain potential effects. Journal of Experimental Psychology: Learning, Memory and Cognition. 22, 1219-1248.

Frisch, S., Kotz, S. A., Von Cramon, D. Y., Friederici, A. D., 2003. Why the P600 is not just a P300: The role of the basal ganglia. Clinical neurophysiology 114, 336-340.

Gabrieli, J. D. E., Poldrack, R. A., Desmond, J. E., 1998. The role of the left pre-frontal cortex in language and memory. Proceedings of the National Academy of Sciences of the United States of America 95, 906-913.

Gunter, T. C., Stowe, L. A., Mulder, G., 1997. When syntax meets semantics. Psychophysiology 34, 660-676.

Haenschel, C., Vernon, D. J., Dwivedi, P., Gruzelier, J. H., Baldeweg, T., 2005. Event-related brain potential correlates of human auditory sensory memorytrace formation. The Journal of Neuroscience 25, 10494-10501.

Hagoort, P., Hald, L., Bastiaansen, M., Petersson, K. M., 2004. Integration of word meaning and world knowledge in language comprehension. Science 304, $438-441$.

Heike, A. E., 1981. A content-processing view of hesitation phenomena. Language and Speech 24, 147-160.

Holcomb, P. J., Neville, H. J., 1991. Natural speech processing: An analysis using event-related potentials. Psychobiology 19, 286-300.

Howell, P., Sackin, S., 2001. Function word repetitions emerge when speakers are operantly conditioned to reduce frequency of silent pauses. Journal of Psycholinguistic Research 30, 457-474. 
Jasper, H. H., 1958. Report to the committee on methods of clinical examination in electroencephalography. Appendix: The ten-twenty system of the international federation. Electroencephalography and Clinical Neurophysiology 10, $370-375$.

Kolk, H., Chwilla, D. J., van Herten, M., Oor, P. J. W., 2003. Structure and limited capacity in verbal working memory: A study with event-related potentials. Brain and Language 85, 1-36.

Kuperberg, G. R., 2007. Neural mechanisms of language comprehension: Challenges to syntax. Brain Research 1146, 23-49.

Kutas, M., Van Petten, C. K., Kluender, R., 2006. Psycholinguistics electrified II (1994-2005). In: Traxler, M., Gernsbacher, M. A. (Eds.), Handbook of Psycholinguistics, 2nd Edition. Elsevier Press, San Diego, CA, Ch. Psycholinguistics electrified II (1994-2005), pp. 83-143.

Ledoux, K., Traxler, M. J., Swaab, T. Y., 2007. Syntactic priming in comprehension: Evidence from event-related potentials. Psychological Science 18, $135-143$.

Maclay, H., Osgood, C. E., 1959. Hesitation phenomena in English speech. Word $15,19-44$.

McCarthy, G., Wood, C. C., 1985. Scalp distributions of event-related potentials: An ambiguity associated with analysis of variance models. Electroencephalography and Clinical Neurophysiology 62, 203-208.

Moreno, E. M., Federmeier, K. D., Kutas, M., 2002. Switching languages, switching palabras (words): An electrophysiological study of code switching. Brain and Language 80, 188-207.

Nagy, M. E., Rugg, M. D., 1989. Modulation of event-related potentials by word repetition: The effects of inter-item lag. Psychophysiology 26, 431-436. 
Okita, T., Jibu, T., 1998. Selective attention and N400 attenuation with spoken word repetition. Psychophysiology 35, 260-271.

Osterhout, L., Hagoort, P., 1999. A superficial resemblance does not necessarily mean you are part of the family: Counterarguments to Coulson, King and Kutas (1998) in the P600/SPS-P300 debate. Language and Cognitive Processes $14,1-14$.

Ranganath, C., Paller, K. A., 1999. Frontal brain activity during episodic and semantic retrieval: Insights from event-related potentials. Journal of Cognitive Neuroscience 11, 598-609.

Rhodes, S. M., Donaldson, D. I., 2008. Association and not semantic relationships elicit the N400 effect: Electrophysiological evidence from an explicit language comprehension task. Psychophysiology 44, 50-59.

Rugg, M. D., 1985. The effects of semantic priming and word repetition on event-related potentials. Psychophysiology 22, 642-647.

Rugg, M. D., Allan, K., Birch, C. S., 2000. Electrophysiological evidence for the modulation of retrieval orientation by depth of study processing. Journal of Cognitive Neuroscience 12, 664-678.

Salmon, N., Pratt, H., 2002. A comparison of sentence- and discourse-level semantic processing: An ERP study. Brain and Language 83, 367-383.

Senkfor, A. J., Van Petten, C. M., 1998. Who said what? An event-related potential investigation of source and item memory. Journal of Experimental Psychology: Learning, Memory, and Cognition 24, 1005-1025.

Shriberg, E. S., August 1995. Acoustic properties of disfluent repetitions. In: Proceedings of the International Congress of Phonetic Sciences. Vol. 4. Stockholm, Sweden, pp. 384-387.

Swaab, T. Y., Camblin, C. C., Gordon, P. C., 2004. Electrophysiological evidence for reversed lexical repetition effects in language processing. Journal Of Cognitive Neuroscience 16 (5), 715-726. 
Van Berkum, J. J. A., Brown, C. M., Hagoort, P., 1999. Early referential context effects in sentence processing: Evidence from event-related brain potentials. Journal of Memory and Language 41, 147-182.

Van Berkum, J. J. A., Brown, C. M., Hagoort, P., Zwitserlood, P., 2003. Eventrelated brain potentials reflect discourse-referential ambiguity in spoken language comprehension. Psychophysiology 40 (2), 235-248.

Van den Brink, D., Brown, C. M., Hagoort, P., 2001. Electrophysiological evidence for early contextual influences during spoken-word recognition: N200 versus N400 effects. Journal of Cognitive Neuroscience 13, 967-985.

Van Herten, M., Kolk, H. H. J., Chwilla, D. J., 2005. An ERP study of P600 effects elicited by semantic reversal anomalies. Cognitive Brain Research 22, $241-255$.

Van Herten, M., Kolk, H. H. J., Chwilla, D. J., 2006. When heuristics clash with parsing routines: ERP evidence for conflict monitoring in sentence perception. Journal of Cognitive Neuroscience 18 (2), 1-17.

Van Petten, C. M., Kutas, M., 1991. Influences of semantic and syntactic context on open and closed class words. Memory and Cognition 19, 95-112.

Van Petten, C. M., Kutas, M., Kluender, R., Mitchiner, M., McIsaac, H., 1991. Fractionating the word repetition effect with event-related potentials. Journal of Cognitive Neuroscience 3, 131-150.

Vissers, C. T. W. M., Chwilla, D. J., Kolk, H. H. J., 2006. Monitoring in language perception: The effect of misspellings of words in highly constrained sentences. Brain Research 1, 150-163. 


\title{
Not all disfluencies are are equal: The effects of disfluent repetitions on language comprehension
}

\author{
Lucy J. MacGregor ${ }^{* a}$, Martin Corley ${ }^{\mathrm{b}}$, David I. Donaldson ${ }^{\mathrm{a}}$ \\ ${ }^{a}$ Department of Psychology, University of Stirling \\ ${ }^{b}$ Psychology, PPLS, University of Edinburgh
}

\begin{abstract}
Disfluencies can affect language comprehension, but to date, most studies have focused on disfluent pauses such as er. We investigated whether disfluent repetitions in speech have discernible effects on listeners during language comprehension, and whether repetitions affect the linguistic processing of subsequent words in speech in ways which have been previously observed with ers. We used eventrelated potentials (ERPs) to measure participants' neural responses to disfluent repetitions of words relative to acoustically identical words in fluent contexts, as well as to unpredictable and predictable words that occurred immediately postdisfluency and in fluent utterances. We additionally measured participants' recognition memories for the predictable and unpredictable words. Repetitions elicited an early onsetting relative positivity (100-400ms post-stimulus), clearly demonstrating listeners' sensitivity to the presence of disfluent repetitions. Unpredictable words elicited an N400 effect. Importantly, there was no evidence that this effect, thought to reflect the difficulty of semantically integrating unpredictable compared to predictable words, differed quantitatively between fluent and disfluent utterances. Furthermore there was no evidence that the memorability of words was affected by the presence of a preceding repetition. These findings contrast with previous research which demonstrated an N400 attenuation of, and an increase in memorability for, words that were preceded by an er.
\end{abstract}

* Corresponding author. Address: Psychological Imaging Laboratory, Department of Psychology, University of Stirling, Stirling, FK9 4LA, UK.

Email address: Lucy.MacGregor@gmail.com (Lucy J. MacGregor) 
However, in a later (600-900ms) time window, unpredictable words following a repetition elicited a relative positivity. Reanalysis of previous data confirmed the presence of a similar effect following an er. The effect may reflect difficulties in resuming linguistic processing following any disruption to speech.

Key words: Language Comprehension, Disfluency, Speech, ERPs, Repetitions

\section{Introduction}

Speakers are rarely fully fluent, but produce speech which is peppered with pauses, ums and ers, and prolonged or repeated words. These disfluencies, which typically occur when the speaker is uncertain how to continue, form part of the linguistic input which a listener must interpret. Although a number of recent studies have used different measures to demonstrate that disfluencies directly affect the comprehension process, the focus of the majority of these studies has been on disfluent pauses, particularly er (or uh).

Ers can speed up response times to subsequent target words in word monitoring (Fox Tree, 2001) or object selection tasks (Brennan and Schober, 2001); further, they can lead listeners to predict the upcoming mention of an item deemed less accessible for the speaker from a constrained set of referents (Arnold, Tanenhaus, Altmann, and Fagnano, 2004; Arnold, Hudson Kam, and Tanenhaus, 2007); and they can also affect the ease of semantic integration of subsequent words (Corley, MacGregor, and Donaldson, 2007). Importantly, ers have been shown to affect not only the process of comprehension but its outcome: In offline studies, Bailey and Ferreira (2003) have demonstrated that ers can affect listeners' interpretations of syntactically ambiguous sentences. Hearing a sentence including an er increases the memorability of the subsequent word (Corley et al., 2007), possibly because of an increase in attention (Collard, Corley, MacGregor, and Donaldson, 2008).

Despite the growing evidence for the effects of ers, few researchers have investigated whether other disfluencies have observable effects on the language comprehension system. The present paper focuses on the disfluent repetition 
of words in an utterance. Disfluent repetitions have been observed to occur in similar situations to disfluent pauses (Beattie and Bradbury, 1979; Howell and Sackin, 2001), at a rate of around 1.5 repetitions per 100 words (Bortfeld, Leon, Bloom, Schober, and Brennan, 2001), with the majority of repetitions comprising function words (2.5 per 100 words: Clark and Wasow, 1998). However, repetitions differ from ers in one very important respect. Ers are clearly distinguishable from the propositional content of an utterance on the basis of their phonology (whether or not they are words: Clark and Fox Tree, 2002). In contrast, a repeated word is recognised as disfluent because of its context.

From the listener's perspective, repetition disfluencies raise two interesting questions. The first concerns the recognition of disfluency: Are there discernible effects on listeners of encountering repeated words which are only disfluent by virtue of their context of occurrence? The second concerns the generality of the reported effects of disfluency: Do repeated words affect the comprehension processes in the same way as ers? Like ers, they introduce time into the speech signal, which is associated with difficulty in aspects of speech production including syntactic planning and lexical retrieval (Blackmer and Mitton, 1991; Clark and Wasow, 1998; Maclay and Osgood, 1959). One possibility is that ers and repetitions are both simple consequences of the speaker's difficulties in planning. For example, Blackmer and Mitton (1991) attribute repetitions at the beginning of phonological phrases to an "autonomous restart capability" within the articulator, according to which existing speech plans are restarted when new material fails to arrive in time. If differing disfluencies reflect similar circumstances in speech, we might expect listeners to treat them equivalently. An alternative view is that some repetitions are used by speakers to establish continuity upon resumption of speaking, in contrast to ers which tend to mark the suspension point (Clark and Wasow, 1998). If listeners are sensitive to such a distinction, we might expect different disfluencies to have different consequences.

To answer these questions, we report an experiment based on previous work by Corley et al. (2007). We use event-related potentials (ERPs) to measure participants' neural responses to disfluent repetitions of words, as well as to the 
words that occur immediately post-disfluency. To establish whether repetition disfluencies have consequences for the outcome of this process, we additionally measure participants' recognition memories for the post-disfluent words. Based on earlier work (Collard et al., 2008; Corley et al., 2007), we anticipate that any attentional changes caused by the disfluent repetitions would enhance memory encoding, ultimately resulting in the post-disfluency being better remembered in a subsequent recognition test.

ERPs are well suited to investigations of speech processing because they can provide a continuous record of brain activity while participants are engaged in comprehension. A number of studies have used ERPs to show observable effects of processing repeated words that do not render the stimuli disfluent. Relative to the first occurrence of a word, repeated words in lists and sentences are commonly associated with an attenuation of the N400 (Besson, Kutas, and Van Petten, 1992; Besson and Kutas, 1993; Ledoux, Traxler, and Swaab, 2007; Okita and Jibu, 1998; Rugg, 1985). The effect is particularly clear if the repeated word is presented immediately after its first occurrence (Nagy and Rugg, 1989). Consistent with the predominant interpretation of the N400 as indicating semantic integration difficulty (e.g., Federmeier, Wlotko, De Ochoa-Dewald, and Kutas, 2007; Hagoort, Hald, Bastiaansen, and Petersson, 2004; Van Berkum, Brown, and Hagoort, 1999; Van Berkum, Brown, Hagoort, and Zwitserlood, 2003; Van Petten and Kutas, 1991; for evidence that the N400 is driven by word associations rather than semantic features, see Rhodes and Donaldson, 2008) these studies suggest that second or later mentions of a word are easier to integrate. Importantly, as would be predicted by models of language comprehension, the presence of a discourse context which renders repeated words unpredictable or unnatural can reverse the N400 attenuation. One example of such a reversal is where the repeated words are definite expressions which co-refer (such as Matt went swimming after Matt had dinner). In these cases there is an increase in the N400 for repeated words (Matt) relative to pronoun controls (he), suggesting that there is greater integration difficulty in cases where pronouns would be predicted (Swaab, Camblin, and Gordon, 2004). Clearly, repetition does affect 
linguistic processing, but studies demonstrate that the effects of repeated words are determined by the context in which they occur. Moreover, to date, we do not know of any studies which have focused on the effects of repeated words in spoken language contexts which render them disfluent.

The studies discussed above all focused on the repeated word itself. In another study of repetitions, Fox Tree (1995) instead investigated the effects of the word which occurs immediately following a disfluent repetition. In a word monitoring task, response times to target words which were preceded by repetitions were no slower than those which were preceded by pauses of equivalent length, and in two experiments, they were faster. One interpretation of these findings is that attention was engaged by the repetitions, leading to faster response times, as has been claimed in the case of er (Fox Tree, 2001). However, a plausible alternative explanation is that pauses in the control condition disrupted processing, resulting in slower response times for this condition relative to the repetition condition. Thus evidence for an effect of repetitions on linguistic processing remains equivocal. Moreover, the task for participants was to monitoring for specific words rather than listen for comprehension, which may have affected the outcome of the experiment. In sum, it is entirely possible that repetition disfluencies affect the processing of the words which follow, and the present study provides evidence to support that possibility.

\subsection{The present study}

The present study is based on an experiment reported in Corley et al. (2007). In the earlier experiment, ERPs were recorded as participants listened to utterances which ended in either predictable or unpredictable target words. Half of the utterances were disfluent by virtue of an er occurring immediately prior to the target word. The results showed that that the presence of an er reduced the amplitude of the N400 effect for unpredictable compared to predictable words. An additional recognition memory test was used to show that target words which had been preceded by er during comprehension were better recognised than those which had not. 
In the current experiment, we focus on repetition disfluencies. The disfluent utterances from the previous experiment were discarded, and novel disfluent utterances were created which contained a repetition immediately preceding the utterance-final target word. Table 1 shows an example stimulus set.

Table 1: Example stimulus set comprising two highly constraining sentence frames, crossed with two utterance-final target words, which were predictable or unpredictable in context. Target words are shown in bold. Half the utterances were disfluent and contained a repetition before the target word, indicated in square brackets.

\begin{tabular}{llll}
\hline Predictable & Everyone's got bad habits and mine is biting my & {$[\mathrm{my}]$} & nails \\
& That drink's too hot; I've just burnt my & {$[\mathrm{my}]$} & tongue \\
Unpredictable & Everyone's got bad habits and mine is biting my & {$[\mathrm{my}]$} & tongue \\
& That drink's too hot; I've just burnt my & {$[\mathrm{my}]$} & nails \\
\hline
\end{tabular}

Given the experimental design, the only factor that rendered repeated tokens disfluent was the preceding context. We were therefore able to compare the ERPs elicited in response to repetition disfluencies with those associated with acoustically identical control words. Since the words were repeated immediately (as in Nagy and Rugg, 1989) but their occurrence was not predictable (as in Swaab et al., 2004), the nature of any effect of repetition under these conditions was one empirical question of considerable interest.

Our second interest concerned the effects of repetition disfluencies on the comprehension of words which occurred later in the utterance. Our predictions were based on the premise that repetitions and er disfluencies have similar distributions in speech (Beattie and Bradbury, 1979; Howell and Sackin, 2001), and thus we expected that the ease with which the post-disfluent target word was integrated would be affected by its predictability (as in Corley et al., 2007). Because disfluency tends to precede less predictable items in speech (Beattie and Butterworth, 1979) we hypothesised that the semantic integration disadvantage for unpredictable items would diminish post-disfluency, resulting in a smaller 
N400 difference between unpredictable and predictable target words following repetitions. Because disfluency affects attention (Collard et al., 2008) we expected target words to be more likely to be recognised if they had been initially encountered post-disfluency (Collard et al., 2008; Corley et al., 2007).

\section{Method}

\subsection{Participants}

Sixteen native British English speakers (7 male; mean age 22; range 1935; all right-handed) who reported no hearing or reading difficulties, and had no known neurological impairment, participated for financial compensation or course credit. Informed consent was obtained in accordance with the University of Stirling Psychology Ethics Committee guidelines.

\subsection{Materials}

The stimuli were 160 highly constrained fluent and disfluent utterances ending in predictable (cloze probability 0.84 , range $0.52-1$ ) or unpredictable (cloze probability 0) target words and were based on those used in Corley et al. (2007). Utterances were constructed in pairs such that each predictable word also served as an unpredictable word for a corresponding utterance. Furthermore, predictable and unpredictable targets completed fluent and disfluent utterances so that across participants each target appeared in every condition. This double counterbalancing ensured that targets were perfectly controlled for grammatical class, duration, frequency, imageability, and concreteness and meant that each participant heard all sentence frames and target words once only. Table 1 shows an example material set.

Stimuli were digitally recorded by a female native English speaker at a natural speaking rate. For each utterance the utterance-final word was replaced by the pseudotarget word pen which meant that there were no acoustic cues to the upcoming word. Any prosodic cues to an upcoming $p$ would have been constant across conditions. Following recording, pseudotargets were excised and replaced 
by target words which had been recorded as utterance-final words in separate carrier sentences.

Disfluent utterances were created from fluent utterances by copying the pretarget word and splicing it into the speech stream, after the original, to form a repetition before the utterance-final target word. Thus each disfluent utterance was identical to a fluent counterpart up to the point of the repeated word. The repetition was typically of a single function word (e.g., the or a) but sometimes more words were repeated (e.g., to the, for the) when this made it easier to obtain utterances without obvious splicing points. A pause of $200 \mathrm{~ms}$ was inserted between the two tokens of the repetition. The duration chosen was based on the pauses which have been observed during naturally occurring repetitions (Fox Tree, 1995, p. 724) and was shortened or lengthened where this resulted, in the experimenters' opinions, in a better sounding recording. No additional silence other than any which existed in the fluent utterance was inserted after the repeated token.

An additional 80 filler utterances of varying constraint were recorded. Forty were fluent and 40 contained disfluencies of various types (repetitions, ers, silent pauses, and repairs) in various locations.

Before presentation, all stimuli were converted to 16-bit $22050 \mathrm{~Hz}$.wav files, and their amplitudes were normalised so that the acoustic volume was approximately matched across stimuli. Four versions of the experiment were created, for counterbalancing purposes, each containing 160 experimental utterances (40 each of fluent predictable, fluent unpredictable, disfluent predictable, and disfluent unpredictable) together with the 80 filler utterances.

\subsection{Procedure}

There were two parts to the experiment. In the first part, participants were told that they would hear a series of utterances which were re-recorded excerpts from natural conversations. Participants were further advised that because the utterances would be heard out of context, some would make more sense than others. They were instructed to listen for understanding, just as they would in 
a natural situation. There was no other task. To minimise the introduction of artifacts into the EEG recording, it was emphasised to participants that they should relax, keep as still as possible, and fixate their eyes on a cross in the centre of the screen.

One hundred and sixty experimental utterances were presented auditorily, in a random order, interspersed with fillers. Utterances were presented in two blocks lasting approximately 15 minutes each, separated by a break of a few minutes. The start of each utterance was indicated visually (for $250 \mathrm{~ms}$ ) by a yellow fixation cross on a black screen, which flashed blue once (for 250ms) and returned to yellow as the utterance began. The fixation cross remained on the screen for the duration of the utterance to discourage eye movements. Following each utterance the screen was blanked for $1500 \mathrm{~ms}$.

Following the first part of the experiment, participants took part in a surprise recognition memory test for the utterance final 'old' words. These words had been either contextually predictable or unpredictable, and had been heard in either fluent or disfluent contexts. They were interspersed with 160 frequencymatched 'new' foils, which had not been heard at any point in the first part of the experiment. Targets were presented visually, and participants discriminated between old and new words as accurately as possible by pressing one of two response keys with index fingers (counterbalanced across participants). The start of each presentation was indicated by the appearance of a fixation cross, which was replaced by the target word. After a $750 \mathrm{~ms}$ presentation, the screen was blanked for $1750 \mathrm{~ms}$.

\subsection{ERP recording and pre-processing}

Electrophysiological data was recorded and analysed in the Psychological Imaging Laboratory at the University of Stirling (http://www.erps.stir .ac. uk) using methods which are standard in the cognitive electrophysiology field. During the first part of the experiment, EEG was recorded (Neuroscan 4.2 Acquire software, Neuromedical Supplies, http://www. neuro.com) from $61 \mathrm{Ag} / \mathrm{AgCl}$ electrodes embedded in an elasticated cap, based on an extended version of the 
international 10-20 system (Jasper, 1958). Data were recorded using a left mastoid reference, and re-referenced offline to the average of left and right mastoid recordings. Electro-oculograms (EOGs) were recorded to monitor for vertical and horizontal eye movements. Electrode impedances were kept below $5 \mathrm{k} \Omega$. The analogue EEG and EOG recordings were amplified (band pass filter $0.01-40 \mathrm{~Hz}$ ), and continuously digitised (16 bit) at a sampling frequency of $200 \mathrm{~Hz}$.

Before off-line averaging, the continuous EEG files for each participant were segmented into $1350 \mathrm{~ms}$ epochs, starting $150 \mathrm{~ms}$ before the critical words, and screened for artifacts. Epochs were excluded when any channel became saturated (exceeding $495 \mu \mathrm{V}$ ), when drift (absolute difference in amplitude between the first and last data point of each individual epoch) was greater than $33.75 \mu \mathrm{V}$, or when amplitude on any channel (excluding VEOG) was greater than $75 \mu \mathrm{V}$. A minimum of 16 artefact-free trials was required from each participant, in each condition, to ensure an acceptable signal-to-noise ratio. The screening process resulted in the loss of $36 \%$ of the trials, with no difference between conditions. The effect of eye-blink artifacts was minimised by estimating and correcting their contribution to the ERP waveforms using a regression procedure which involves calculating an average blink from 32 blinks for each participant, and removing the contribution of the blink from all other channels on a point-by-point basis. Waveforms were baseline corrected by subtracting the mean amplitude over the interval preceding the critical word and smoothed over 5 points so that each sampling point represents the average over the two previous and two subsequent points.

Grand average ERPs were formed time-locked to the critical words, in each condition, for each participant, and then averaged over all participants. ERPs were quantified by measuring the mean amplitude over time windows of interest, for each electrode, in each condition, for each participant. Quantitative differences between conditions were assessed using analyses of variance (ANOVAs). Differences in scalp distributions of effects between conditions were assessed after normalisation for amplitude differences using the Max/Min method (McCarthy and Wood, 1985). All analyses made use of Greenhouse-Geisser corrections 
where appropriate, and are reported using corrected $F$ and $p$ values.

\section{Results}

The results are presented in three sections: First, we consider the effects at the repetition itself, second, we consider the effects at the post-repetition target word, and finally we consider the memory results.

\subsection{Effects at the repetition}

Based on visual inspection of the waveforms, ERPs were quantified by measuring the mean amplitude of the ERP difference between repetition and control words over 100-400ms. Initially, the effects were also analysed over shorter time windows of $50-150 \mathrm{~ms}$ and $150-400 \mathrm{~ms}$, but no differences between these time windows were observed, and the results reported here are from the $100-400 \mathrm{~ms}$ time window only. The effects were not analysed after $400 \mathrm{~ms}$ because of the potential overlap with the effects time-locked to the utterance-final words, which are analysed separately below.

The repetition effect was assessed by forming grand average ERPs timelocked to the repetitions and comparing them to ERPs formed to (acoustically identical) corresponding control words in fluent utterances. Importantly, in an ANOVA with factors of Predictability [predictable, unpredictable], Repetition [repetition, control], Location [F, FC, C, CP, P], Hemisphere [left, right], and Site [superior: electrode $1 / 2$, medial: electrode $3 / 4$, inferior: electrode $5 / 6$ ], no interactions involving both repetition and predictability reached significance The lack of any interactions show that, as expected, there was no evidence for different effects of repetition which occurred before either predictable or unpredictable words. Therefore, the data for the repeated and control words are presented here collapsed over the predictable/unpredictable conditions. This resulted in two conditions, repetition and control, both of which had mean trial numbers of 26 .

Figure 1 shows the relevant ERPs for midline and grouped left and right hemisphere electrodes. Relative to fluent control words, repeated words show 
a positivity which is broadly distributed over the scalp, but appears larger and longer-lasting over central sites, with a slight right hemisphere bias. The effect onsets around 50ms, is larger over 100-400ms and continues until around 600ms.
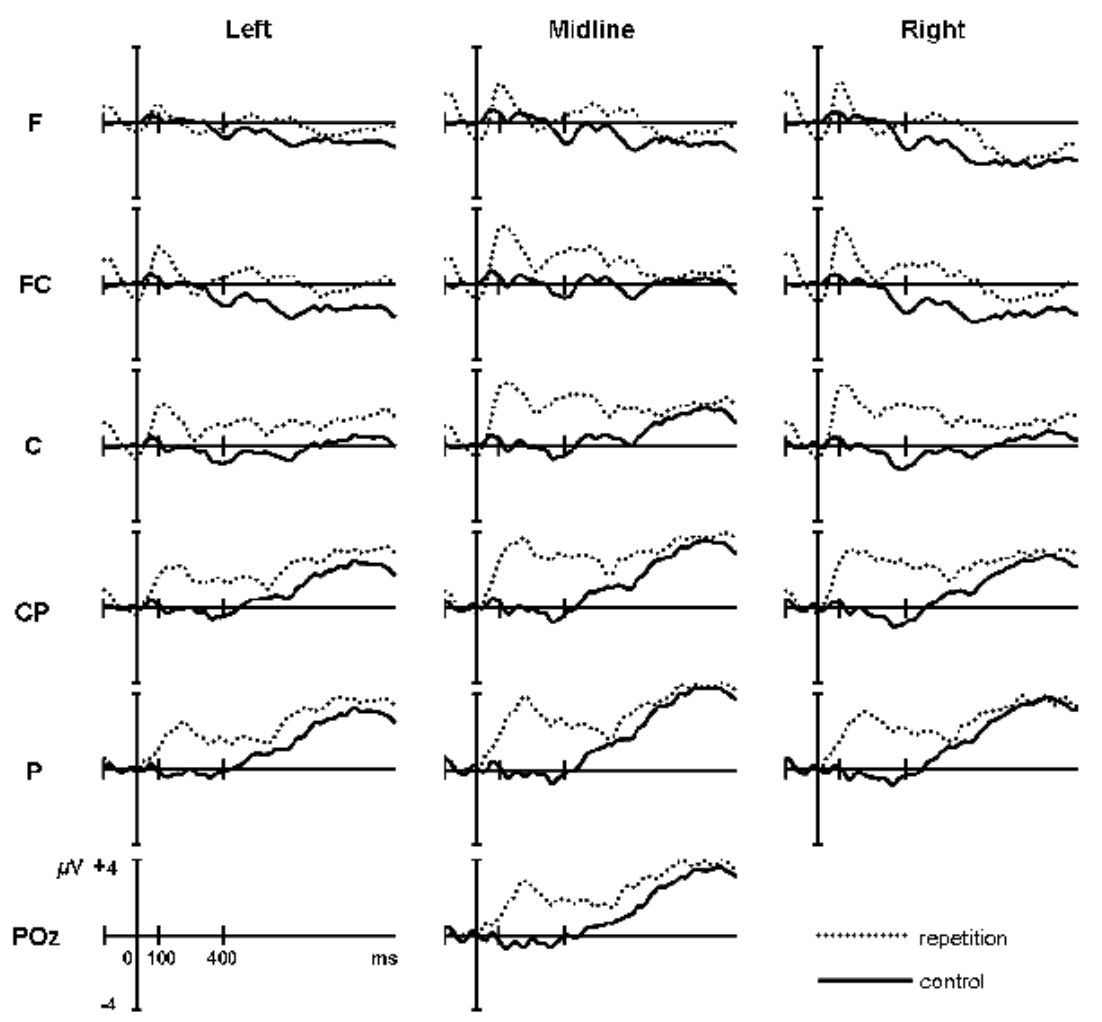

Figure 1: ERPs relative to repeated (dotted lines) or fluent control (dotted lines) word onsets. Positive is plotted up. The central column represents the midline sites (from top: frontal (F), fronto-central (FC), central (C), centro-parietal (CP), parietal (P), occipito-parietal (PO)); the left-hand and right-hand columns represent averages of three electrodes to the left or right of the midline respectively.

The topographic distribution of the repetition effect over 100-400ms is shown in Figure 2.

An ANOVA using the factors of Repetition, Location, Hemisphere, and Site on mean amplitudes over the 100-400ms time window revealed a main effect of repetition $\left[F(1,15)=15.513, \eta_{p}^{2}=.508, p=.001\right]$, reflecting the overall posi- 


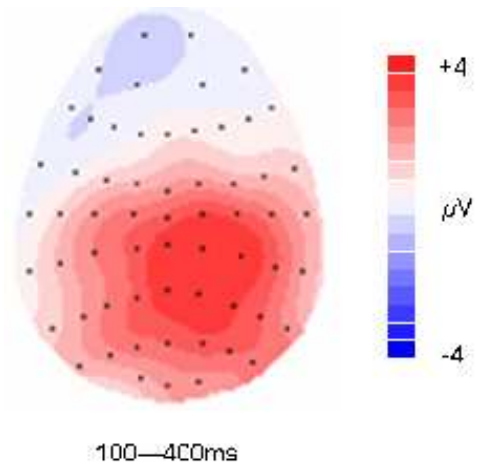

Figure 2: Scalp topography showing the repetition effect over 100-400ms.

tivity for repetitions relative to control words. There were interactions between repetition and location $\left[F(4,60)=24.032, \eta_{p}^{2}=.616, p<.001\right]$, between repetition and hemisphere $\left[F(1,15)=11.109, \eta_{p}^{2}=.425, p=.005\right]$, and between repetition and site $\left[F(2,30)=20.848, \eta_{p}^{2}=.582, p<.001\right]$, reflecting the larger positivity at central/centro-parietal locations, over the right hemisphere, and at superior sites. There was also a three-way interaction between repetition, location and site $\left[F(8,120)=4.690, \eta_{p}^{2}=.238, p=.009\right]$ reflecting a larger positivity over superior sites, at central/centro-parietal locations.

\subsection{Effects at the post-disfluency target}

Effects post-repetition were assessed using grand average ERPs time-locked to the onsets of the utterance-final predictable and unpredictable words. These were created separately for fluent and disfluent utterances, resulting in four conditions: fluent predictable, fluent unpredictable, disfluent predictable, disfluent unpredictable, with mean trial numbers of $26,26,25$, and 26 respectively.

Figure 3 shows relevant ERPs for fluent and disfluent utterances, for midline and grouped left and right hemisphere electrodes. Relative to predictable words, unpredictable words show a negativity over the $300-500 \mathrm{~ms}$ time window which is broadly distributed over the scalp, but appears larger over centroparietal/parietal and midline sites. Although the morphology of the individual 
waveforms is different for fluent and disfluent utterances, similar differences between predictable and unpredictable conditions are evident in each case. The timing and topography of these differences are compatible with their identification as N400 effects (which tend to onset earlier under conditions of auditory presentation: e.g., Connolly and Phillips, 1994; Holcomb and Neville, 1991; Van den Brink, Brown, and Hagoort, 2001).

Following the N400, differences emerge between fluent and disfluent utterances. For fluent utterances, the relative negativity for unpredictable words continues, but appears smaller and more focused at central sites. By contrast, for disfluent utterances, unpredictable words show a relative positivity over frontal and fronto-central sites bilaterally, and over left centro-parietal/parietal sites.

ERPs were quantified over two time windows: the standard N400 time window (300-500ms) and a later (600-900ms) time window based on inspection of the waveforms. Topographic distributions of the effects for fluent and disfluent utterances over the two time windows can be found in Figure 5 .

The effect of predictability was assessed first in an omnibus ANOVA with the factors of Fluency [fluent, repetition], Predictability [predictable, unpredictable], Location [F, FC, C, CP, P], Hemisphere [left, right], and Site [superior: electrode $1 / 2$, medial: electrode $3 / 4$, inferior: electrode 5/6]. Subsequent analyses concentrated on the effects for fluent and disfluent conditions separately. When no effects involving hemisphere were found, further analyses concentrated on midline electrodes, using the factors of Predictability [predictable, unpredictable] and Location $[\mathrm{Fz}, \mathrm{FCz}, \mathrm{Cz}, \mathrm{CPz}, \mathrm{Pz}, \mathrm{POz}]$.

To evaluate the difference in the scalp distributions of the predictability effects between fluent and disfluent conditions, the data were additionally normalised and analysed using ANOVAs with the factors of Fluency [fluent, disfluent] and Site [61 electrodes] or Fluency [fluent, disfluent] and Location [Fz, FCz, $\mathrm{Cz}, \mathrm{CPz}, \mathrm{Pz}, \mathrm{POz}]$. Differences in scalp distributions of the predictability effects were also evaluated over time for fluent and disfluent conditions separately, using ANOVAs with the factors of Window [early, late] and Site [61 electrodes]. 

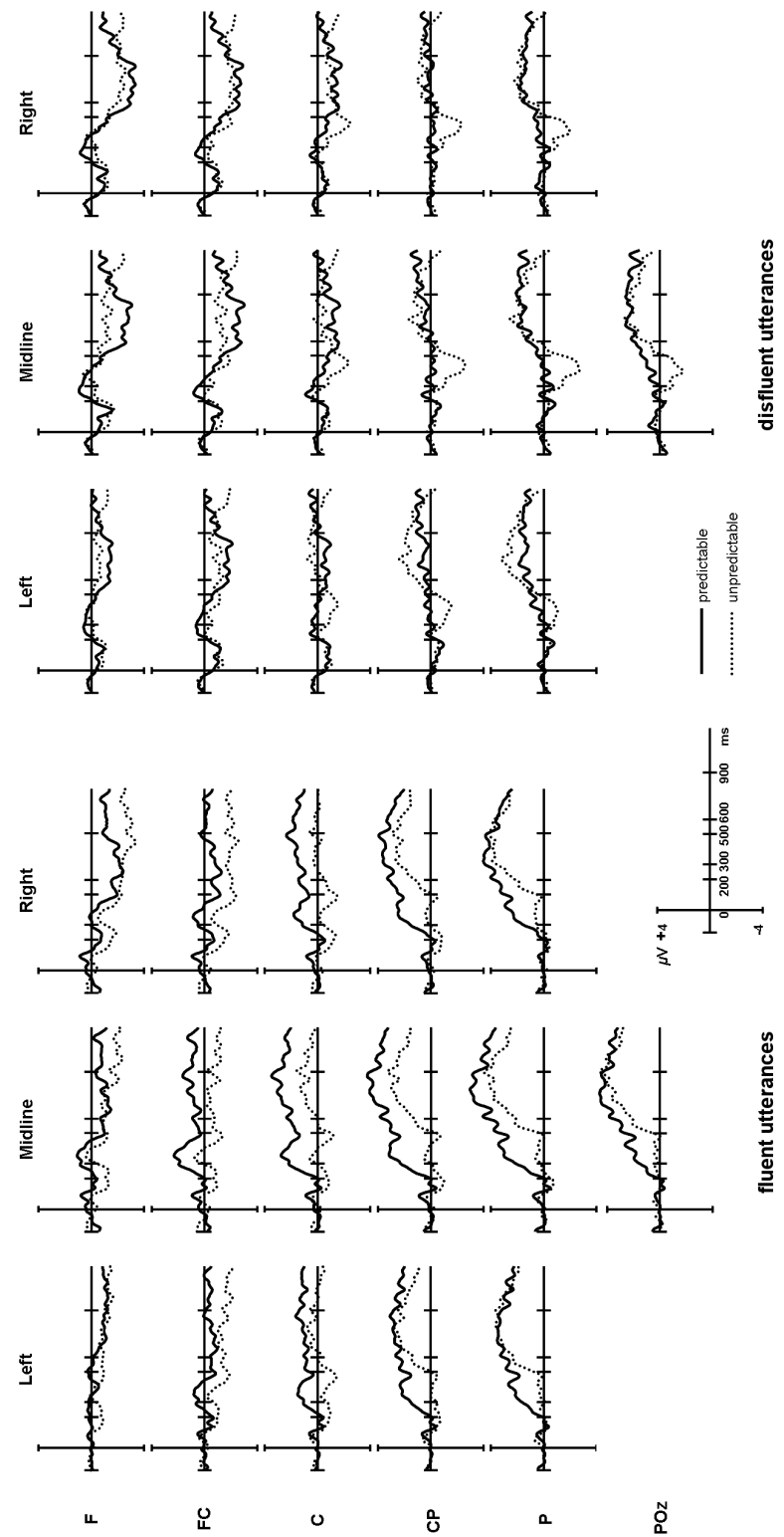

Figure 3: ERPs for fluent (left) and disfluent (right) utterances relative to predictable (solid lines) or unpredictable (dotted lines) target word onsets. Positive is plotted up. The central column of each panel represents the midline sites (from top: frontal ( $\mathrm{F}$ ), fronto-central (FC), central (C), centro-parietal (CP), parietal (P), occipito-parietal (PO)); the left-hand and righthand columns represent averages of three electrodes to the left or right of the midline respectively. 


\subsection{1. $300-500 \mathrm{~ms}$}

Over the 300-500ms time window, an omnibus ANOVA with factors of Fluency, Predictability, Location, Hemisphere and Site showed a a main effect of predictability $\left[F(1,15)=18.16, \eta_{p}^{2}=.557, p=.001\right]$, but no indication of any difference between these effects for fluent and disfluent conditions. Since no effects involving hemisphere were found, a further comparison of the effects for fluent and disfluent conditions was performed at the midline electrodes with the factors of Fluency and Location. Again, there was an expected main effect of predictability $\left[F(1,15)=16.36, \eta_{p}^{2}=.522, p=.001\right]$, reflecting a relative negativity for unpredictable words. There was also a main effect of fluency $\left[F(1,15)=10.15, \eta_{p}^{2}=.404, p=.006\right]$, reflecting the greater overall positivity of the ERPs for fluent utterances. Importantly, there was still no evidence of differences in the effects of predictability between fluent and disfluent conditions.

There was no indication of any differences in the effect of predictability between fluent and disfluent conditions. Because this differed from previous findings using er (Corley et al., 2007), we decided to explore the effects for fluent and disfluent conditions separately, to establish the existence of N400 effects and to compare the topographies of these effects. For fluent utterances there was a main effect of predictability $\left[F(1,15)=17.76, \eta_{p}^{2}=.542, p=.001\right]$ and an interaction between predictability and site $\left[F(2,22)=5.26, \eta_{p}^{2}=.259\right.$, $p=.025]$, reflecting a relative negativity for unpredictable words which was larger towards the midline. For disfluent utterances, there was a main effect of predictability $\left[F(1,15)=6.65, \eta_{p}^{2}=.307, p=.021\right]$ reflecting a relative negativity for unpredictable words, but no other significant effects.

Since no effects involving hemisphere were found for either fluent or disfluent conditions, further analyses focused on effects at the midline electrodes. For fluent utterances, there was a main effect of predictability $[F(1,15)=16.222$, $\left.\eta_{p}^{2}=.520, p=.001\right]$ and an interaction between predictability and location $\left[F(5,75)=4.052, \eta_{p}^{2}=.213, p=.032\right]$, reflecting a relative negativity which was larger at more posterior locations. Disfluent utterances showed a main 
effect of predictability $\left[F(1,15)=5.635, \eta_{p}^{2}=.273, p=.031\right]$, again reflecting a relative negativity.

As a final check that the N400s for fluent and disfluent conditions were equivalent, we performed an ANOVA on the normalised data to assess for topographic differences. There was no evidence of a distributional difference between the effects for fluent and disfluent conditions over the $300-500 \mathrm{~ms}$ time window, neither when data from all electrodes were included $[F \mathrm{~s}<1]$, nor when data from just the midline electrodes were included [ $F \mathrm{~s}<1]$. As there is no evidence that the scalp topographies differ between the fluent and disfluent conditions, there is no reason to suppose that different neural generators are responsible for the recorded effects of predictability. ${ }^{1}$

\subsection{2. $600-900 \mathrm{~ms}$}

Over the $600-900 \mathrm{~ms}$ time window an ANOVA with factors of Fluency, Predictability, Location, Hemisphere, and Site revealed an interaction between fluency and predictability $\left[F(1,15)=6.001, \eta_{p}^{2}=.286, p=.027\right]$, indicating a difference between the predictability effects in fluent and disfluent conditions. There were also interactions between predictability, location and site $\left[F(8,120)=14.001, \eta_{p}^{2}=.483, p<.001\right]$, and a main effect of fluency $[F(1,15)=$ $\left.11.544, \eta_{p}^{2}=.435, p=.004\right]$. A midline ANOVA including factors of Predictability and Fluency also showed an interaction between fluency and predictability $\left[F(1,15)=7.795, \eta_{p}^{2}=.342, p=.014\right]$. The normalised analyses showed no significant effects.

\footnotetext{
${ }^{1}$ We conducted a further set of analyses using a time window enlarged by $40 \%$ over the standard 300-500ms window. An ANOVA using this window (from 260-540ms) including factors of Fluency, Predictability, Hemisphere and Site showed that the results did not differ from those reported above. There was no interaction between fluency and predictability, and other effects were also in line with the previous analysis [main effect of predictability: $F(1,15)=15.468, \eta_{p}^{2}=.508, p=.001$; interaction between predictability and location: $F(4,60)=5.428, \eta_{p}^{2}=.266, p=.027$; interaction between predictability and site: $F(2,30)=$ $\left.4.295, \eta_{p}^{2}=.223, p=.05\right]$. Separate analyses of the fluent and disfluent materials using the extended time window did not differ from those reported above.
} 
Fluent and disfluent conditions were analysed separately, and ANOVAs with the factors of Predictability, Location, Hemisphere, and Site provided no evidence that the distributions of the effects were lateralised in either case. For the fluent condition there were interactions between predictability and site $\left[F(2,30)=16.36, \eta_{p}^{2}=.522, p=.001\right]$, and between predictability, location and site $\left[F(8,120)=7.548, \eta_{p}^{2}=.335, p=.004\right]$, reflecting a larger negativity towards midline sites, and more so at central locations. For disfluent utterances there was an interaction between predictability, location and site $\left[F(8,120)=7.714, \eta_{p}^{2}=.340, p=.002\right]$, reflecting a relative positivity over the frontal location which was larger at superior sites.

\subsubsection{Effects over time}

Finally, we investigated the distributions of effects over time, separately for fluent and disfluent stimuli. ANOVAs performed on normalised data with the factors of Window and Site provided no evidence of distributional differences between the effects over the $300-500 \mathrm{~ms}$ and $600-900 \mathrm{~ms}$ time windows for fluent conditions. By contrast, for disfluent conditions there was a significant interaction between Window and Site $\left[F(60,600)=2.952, \eta_{p}^{2}=.164, p=.032\right]$, suggesting a distributional difference between the effects over the two time windows, in particular at midline sites. Specifically, a negative-going effect in the earlier time window develops into a positive-going effect in the later time window.

\subsection{Memory performance}

Memory performance was quantified as the probability of correctly identifying old (previously heard) words. To control for differences in individual memory performance, we treated stimulus identity as a random factor. ${ }^{2}$

\footnotetext{
${ }^{2}$ Traditional adjustments for individual error-rates, such as $d^{\prime}$, are inappropriate here, since the properties of 'old' stimuli are determined by their context of occurrence and hence there are no comparable categories of 'new' stimuli. Using stimulus identity as a random factor ensures that per-participant biases to respond "old" or "new" are controlled for across the
} 
Overall, $61 \%$ of the old words were correctly recognised (false alarm rate $22 \%$ ). Figure 4 shows the recognition probability of utterance-final words by fluency and predictability.
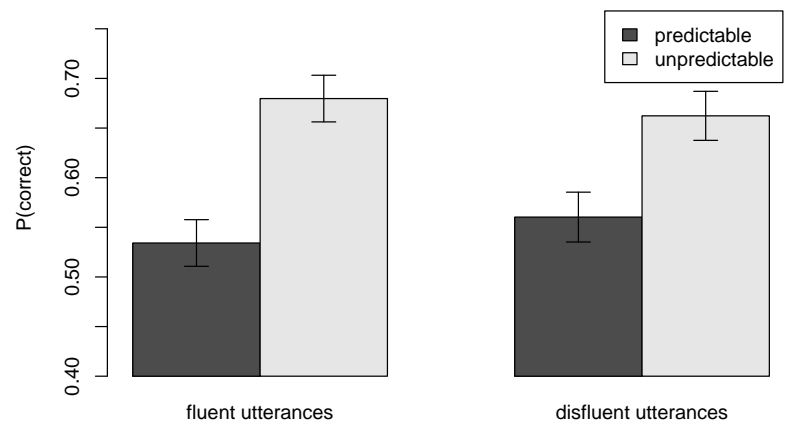

Figure 4: Memory performance for utterance-final words which were originally predictable (black bars) or unpredictable (grey bars) in their contexts, for fluent and disfluent conditions. Error bars represent one standard error of the mean.

An ANOVA carried out with the factors of Fluency [fluent, disfluent] and Predictability [predictable, unpredictable] showed that words that had been unpredictable in their contexts were more likely to be correctly recognised than words that had been predictable $\left[67 \%\right.$ vs. $55 \%: F(1,133)=27.12, \eta_{p}^{2}=.169$, $p<.001]$. This was the case for those which had occurred in fluent [68\% vs. $54 \%$ : $t(133)=4.757, p<.001]$ or in disfluent [66\% vs. $56 \%: F(133)=3.399, p<.001]$ utterances. There were, however, no effects involving disfluency [interaction

experiment.

Twelve target words were inadvertently repeated in the experiment, resulting in 148 distinct targets. Analysis with data from the repeated targets removed did not affect the outcome. One word (party) was never responded to by participants within the allocated time, resulting in no data for this item. Presentation of one other word (garden) was corrupted. These items were excluded from the analyses. 
between disfluency and predictability: $F(1,133)=1.34, \eta_{p}^{2}=.010, p<.249$; main effect of disfluency: $F<1]$.

\section{Discussion}

The present experiment investigated whether listeners are affected by the presence of disfluent repetitions encountered during language comprehension, and whether the processing of words which follow a repetition are affected in the same ways that have been observed previously with ers (Corley et al., 2007). Repetitions elicited a relative positivity in the ERP waveform, reliable over a 100-400ms time window, relative to acoustically identical control words in fluent utterances. This positivity provides clear evidence that listeners were sensitive to the disfluencies they encountered. As expected, unpredictable words elicited a relative negativity compared to predictable words, identifiable as a standard N400 effect. In contrast to ers, which have been associated with an attenuation of the N400 to subsequent words, disfluent repetitions preceding the target words, did not lead to a difference in amplitude (or topography) of the effect in the $300-500 \mathrm{~ms}$ time window. Beyond $500 \mathrm{~ms}$, however, the effects elicited by fluent and disfluent utterances differed. During a later (600-900ms) time window, unpredictable words in fluent utterances showed a relative negativity, which appeared to be a continuation of the N400 observed in the earlier window. By contrast, unpredictable words in disfluent utterances gave rise to a positivity over frontal sites. Although this late positivity shows that there is a difference between processing fluent and disfluent utterance, performance on the recognition task was not affected by disfluency, as would be expected based on the lack of differences in the $300-500 \mathrm{~ms}$ time window. Direct comparisons of the effects of particular types of (predictable or unpredictable) target between fluency conditions are not warranted by the present experimental design, because the pre-stimulus ERP baselines are obtained from sentences which include an extra word in the repetition condition. In the present study this extra word is itself associated with a positivity, making the problem particularly salient. 
The memory results appear to pattern with the post-disfluency results in the $300-500 \mathrm{~ms}$ time window, in that there are no differences attributable to fluency. The equivalent performances in memory across conditions lends credence to the suggestion made by Corley et al. (2007) that their finding of a disfluency advantage in recognition relates to a difference in processing indexed by the N400.

\subsection{Listeners are sensitive to disfluent repetitions}

Hearing a repeated word in a disfluent context resulted in a positive-going shift in the ERP waveform relative to the ERPs formed to acoustically identical control words. This positivity has a similar scalp distribution to the $\mathrm{P} 600$, which has previously been associated with syntactic repair or reanalysis (Friederici, 1995, 2002; Friederici, Hahne, and Mecklinger, 1996; Gunter, Stowe, and Mulder, 1997), and more recently with the resolution of conflict between what has been encountered and what would be predicted based on previous experience (Kolk, Chwilla, van Herten, and Oor, 2003; Van Herten, Kolk, and Chwilla, 2005, 2006; Vissers, Chwilla, and Kolk, 2006; see also Kuperberg, 2007, for an alternative conflict-based mechanism). Since repetitions in the present experiment tend to introduce syntactically illegal sequences (e.g., my my in I've just burnt my my tongue), the antecedent conditions are also compatible with a P600 interpretation. ${ }^{3}$

The repetition-related positivity onsets earlier than is typical for the P600 (early P600 onsets are usually around 200ms: Kutas, Van Petten, and Kluender, 2006). In the present case, the early onset of the effect (approximately 50ms) may reflect the ease of detection of a repetition: Since legal repeated-word sequences are extremely rare, listeners may not need to process the structure of a disfluent repetition in much detail in order to decide that it is illegal. Alter-

\footnotetext{
${ }^{3}$ One continuing suggestion is that the P600 is a member of the P300 family (e.g., Coulson, King, and Kutas, 1998; Gunter et al., 1997), but other evidence suggests a distinction (Frisch, Kotz, Von Cramon, and Friederici, 2003; Osterhout and Hagoort, 1999).
} 
natively, the early onset may be attributable to simple detection of a stimulus repetition, which would result in an overlapping but distinctive earlier positive effect such as the P2, an ERP component related to sensory or perceptual processing of stimuli. Although there is no evidence in the current data, the presence of an early positivity is compatible with previous studies that have shown relative positivities to repeated stimuli. For example, an early positivity (30$250 \mathrm{~ms}$ ) with a bilateral fronto-central distribution has been shown in response to repeated tones (Haenschel, Vernon, Dwivedi, Gruzelier, and Baldeweg, 2005) and this has been linked to sensory memory formation.

While it is not possible to determine the exact functional interpretation in a single experiment, it is clear that listeners quickly detect a repetition disfluency. The ERP component associated with this detection occurs early, suggesting that it may be a sensory response to a repeated word. However, the distribution is posterior and the effect is long-lasting, similar to later-occurring P600 effects which index the effects of linguistic or memory-based systems. A speculation would be that listeners are sensitive to both sensory and linguistic properties of repetitions, and that the ERP reflects the interaction between exogenous and endogenous neural generators. The primary consequence of this sensitivity is in the processing of subsequent words, as discussed in section 4.3 below.

\subsection{Disfluent repetitions show different effects to ers on the processing of sub- sequent words}

Repetition disfluencies did not affect the N400 associated with target words or the likelihood of later recognising those words. This contrasts with the case er, where there is clear evidence that the N400, and memory for subsequent words, are affected (Corley et al., 2007). A straightforward interpretation of these findings would suggest that listeners were sensitive to different functions attributed to different types of disfluency, in line with the type of distinction proposed by Clark and Wasow (1998).

Clark and Wasow suggest that repetitions can be used by speakers to serve more than one purpose, and a possible reason that repetition effects are not 
found in the present experiment may lie in a distinction originally proposed by Heike (1981), between repetitions which are followed by silence and those which are not. In line with the majority of observed repetitions (Shriberg, 1995), our materials did not include an additional pause after the repetition. According to Heike, the repeated elements in such cases may mark the resumption of fluent speech following a minor disruption, rather than constituting an interruption in themselves.

From the point of view of the listener, repetitions, even if produced as an automatic consequence of speaker difficulty (e.g., Blackmer and Mitton, 1991), differ from ers in ways which could account for different effects in processing. First, repetitions are lexicalised and are therefore not immediately distinct from the surrounding lexical context, unlike interruptions of debatable lexical status such as ers. An account which focuses on the lexical nature of the disfluency would predict that the effects of other lexical interruptions, for example lexical fillers such as like or y'know, would be similar to those of repetitions. Second, repetitions, unlike pauses, are probably part of the context into which subsequent words are integrated. Although they delay the onset of subsequent new information, conceptualisation of repetitions as a form of 'delay' from the listener's perspective may not be valid, because there is no delay between the repeated word and the subsequent new word. By contrast, ers clearly introduce a delay between the new information and the context into which it must be integrated. An account which attributes the significant effects to the introduction of a delay would predict that the effect of lexical fillers such as like or y'know, would be similar to those of ers.

The potential classification of types of disfluency requires further investigation to determine whether disfluency effects on listeners are lexical or delay oriented, and whether the disfluencies themselves reflect automatic or deliberate speech processes. Indeed it is unclear whether any of these possibilities exclude others. Regardless, it remains clear that repetitions do affect processing, as evidenced by effects on the processing of target words in the later time window. We now turn our attention to the late positivity found for unpredictable words 
when there has been a repetition disfluency.

\subsection{Disfluent repetitions show a similar effect to ers on the processing of sub- sequent words}

The timing, distribution, and antecedent conditions of the late positivity are compatible with its identification as a Late Positive Complex (LPC), a positive deflection in the waveform, occurring approximately 500-900 ms after stimulus onset, which has with a frontal focus and may be more prominent over the left hemisphere. Because this positivity depends on predictability, it is unlikely to reflect any ongoing effect of the repetitions, and we therefore attribute it to the target words. Indeed, the LPC has been observed in conditions often associated with the elicitation of an N400. It has been observed in response to unexpected words completing highly constrained sentences (Federmeier et al., 2007), idioms (Moreno, Federmeier, and Kutas, 2002), or stories (Salmon and Pratt, 2002) relative to the most expected words, and to probe words which are unrelated to preceding jokes (Coulson and $\mathrm{Wu}, 2005$ ) relative to semantically related controls. It is attenuated for repeated words in a sentence context relative to their first presentation (Van Petten, Kutas, Kluender, Mitchiner, and McIsaac, 1991).

Functional interpretations of the LPC are typically related to aspects of memory control. This is largely because the frontal distribution of the LPC is similar to positivities that are observed in studies of memory and are associated with retrieval effort (Ranganath and Paller, 1999; Rugg, Allan, and Birch, 2000), or attempts to retrieve source information from memory (Senkfor and Van Petten, 1998). Furthermore, the distribution of the LPC is consistent with a generator in the left inferior prefrontal cortex (Coulson and Wu, 2005), a brain region which is often activated during memory tasks, particularly those involved in semantic processing (Gabrieli, Poldrack, and Desmond, 1998; for a review, see Buckner, 2003).

The presence of the late relative positivity for unpredictable words in the current study is consistent with the memory control account of the LPC. However, two competing explanations are equally plausible. The effect may be associated 
with retrieval of the preceding context and suppression of semantic information associated with the most predictable word (cf. Federmeier et al., 2007), or processes involved with updating working memory (cf. Van Petten et al., 1991). From a theoretical perspective, memory control processes are likely to be engaged as participants attempt to resume structural and, particularly, semantic interpretation of the message after a suspension in interpretation caused by the interruption. Regardless of which of these interpretations is correct, if the presence of the positivity is dependent on the interruption to speech, we would expect a similar effect to be observed following other disfluencies such as ers. We therefore analysed data from Corley et al. (2007) using the same strategy used for the $600-900 \mathrm{~ms}$ analysis of the repetition data in the current experiment. Figure 5 shows the topographic distributions of the effects for fluent and disfluent utterances which included an er over the 300-500ms and 600-900ms time windows, together with the effects from the present experiment for comparison.

For fluent utterances from Corley et al. (2007), an ANOVA of effects at 600$900 \mathrm{~ms}$ showed a marginal effect of predictability $\left[F(1,11)=4.392, \eta_{p}^{2}=.285\right.$, $p<.060]$, reflecting the fact that the N400 continues, although in a weaker

form, throughout this window. For disfluent utterances, there was a three-way interaction between predictability, location and site $\left[F(8,88)=4.344, \eta_{p}^{2}=.286\right.$, $p=.026]$, reflecting a relative positivity for unpredictable words over the frontal sites close to the midline. As is clear from a comparison of the panels in Figure 5 , this positivity is similar to the effect observed in the present study. Thus unpredictable words elicit a similar late effect following repetitions and ers, compatible with the proposal that the positivity is related to the impact of the disruption.

\section{Conclusions}

It is well known that listeners are sensitive to the presence of disfluencies encountered during comprehension, but the majority of studies to date have focused on the disfluent pause er. The present study focused on repetition 

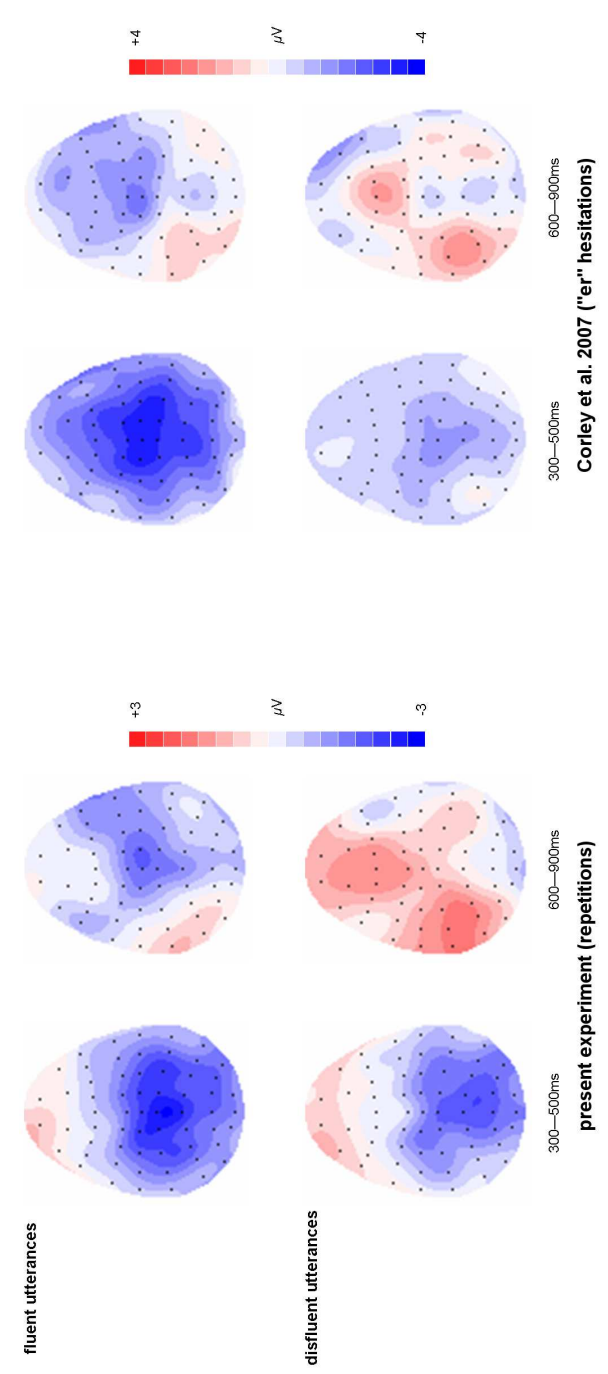

Figure 5: Scalp topographies showing the predictability effects from the present study (left panel) and from Corley et al. (2007) (right panel) over two time windows: 300-500ms and 600-900ms. Data are shown for fluent utterances (top) and for disfluent (bottom) utterances which included either a repetition (left panel) or an er (right panel). 
disfluencies. ERPs revealed that repetitions can be detected within $50 \mathrm{~ms}$ and that following their detection processes of repair and reanalysis are engaged. However, the ERP record provides no evidence that repetitions affect the ease with which subsequent words can be integrated into the discourse as a function of their predictability (as indexed by the magnitude of the N400 effect). This finding stands in stark contrast to the effects of ers observed in previous studies where the N400 effect was attenuated following disfluency, suggesting that, at least from the perspective of the listener, not all disfluencies are equal. Nonetheless, the ERPs revealed an effect of repetitions on the processing of post-disfluent words in a later time window, which may reflect an increase in the difficulty associated with resuming structural and semantic interpretation following an unexpected interruption to an otherwise fluent utterance.

\section{Acknowledgements}

We thank Catriona Bruce for help with data collection and two anonymous reviewers for helpful comments on earlier versions of this paper. The research was partially supported by the Economic and Social Research Council (LJM). DID is a member of the SINAPSE Collaboration (www . sinapse . ac.uk), a Pooling Initiative funded by the Scottish Funding Council and the Chief Scientist Office of the Scottish Executive.

\section{References}

Arnold, J. E., Hudson Kam, C. L., Tanenhaus, M. K., 2007. If you say thee uh you are describing something hard: The on-line attribution of disfluency during reference resolution. Journal of Experimental Psychology: Learning, Memory and Cognition 33, 914-930.

Arnold, J. E., Tanenhaus, M. K., Altmann, R. J., Fagnano, M., 2004. The old and thee, uh, new. disfluency and reference resolution. Psychological Science $15,578-582$. 
Bailey, K. G. D., Ferreira, F., 2003. Disfluencies affect the parsing of gardenpath sentences. Journal of Memory and Language 49, 183-200.

Beattie, G. W., Bradbury, R. J., 1979. An experimental investigation of the modifiability of the temporal structure of spontaneous speech. Journal of Psycholinguistic Research 8, 225-248.

Beattie, G. W., Butterworth, B. L., 1979. Contextual probability and word frequency as determinants of pauses and errors in spontaneous speech. Language and Speech 22, 201-211.

Besson, M., Kutas, M., 1993. The many facets of repetition: A cued-recall and event-related potential analysis of repeating words in the same versus different sentence contexts. Journal of Experimental Psychology: Learning, Memory, and Cognition 19, 1115-1133.

Besson, M., Kutas, M., Van Petten, C., 1992. An event-related potential (ERP) analysis of semantic congruity and repetition effects in sentences. Journal of Cognitive Neuroscience 4, 132-149.

Blackmer, E. R., Mitton, J. L., 1991. Theories of monitoring and the timing of repairs in spontaneous speech. Cognition 39, 173-94.

Bortfeld, H., Leon, S. D., Bloom, J. E., Schober, M. F., Brennan, S. E., 2001. Disfluency rates in conversation: Effects of age, relationship, topic, role, and gender. Language and Speech 44, 123-147.

Brennan, S. E., Schober, M. F., 2001. How listeners compensate for disfluencies in spontaneous speech. Journal of Memory and Language 44, 274-296.

Buckner, R. L., 2003. Functional-anatomic correlates of control processes in memory. Journal of Cognitive Neuroscience 23, 3999-4004.

Clark, H. H., Fox Tree, J. E., 2002. Using uh and um in spontaneous speaking. Cognition 84, 73-111. 
Clark, H. H., Wasow, T., 1998. Repeating words in spontaneous speech. Cognitive Psychology 37, 368-407.

Collard, P., Corley, M., MacGregor, L. J., Donaldson, D. I., 2008. Attention orienting effects of hesitations in speech: Evidence from ERPs. Journal of Experimental Psychology: Learning, Memory and Cognition 34, 696-702.

Connolly, J. F., Phillips, N. A., 1994. Event-related potential components reflect phonological and semantic processing of the terminal word of spoken sentences. Journal of Cognitive Neuroscience 6, 256-266.

Corley, M., MacGregor, L. J., Donaldson, D. I., 2007. It's the way that you, er, say it: Hesitations in speech affect language comprehension. Cognition 105, 658-668.

Coulson, S., King, J. W., Kutas, M., 1998. Expect the unexpected: Eventrelated brain response to morphosyntactic violations. Language and Cognitive Processes 13, 21-58.

Coulson, S., Wu, Y. C., 2005. Right hemisphere activation of joke-related information: An event-related brain potential study. Journal of Cognitive Neuroscience $17,494-506$.

Federmeier, K. D., Wlotko, E. W., De Ochoa-Dewald, E., Kutas, M., 2007. Multiple effects of sentential constraint on word processing. Brain Research $1146,75-84$.

Fox Tree, J. E., 1995. The effects of false starts and repetitions on the processing of subsequent words in spontaneous speech. Journal of Memory and Language 34, 709-738.

Fox Tree, J. E., 2001. Listeners' uses of um and uh in speech comprehension. Memory and Cognition 29, 320-326.

Friederici, A. D., 1995. The time course of syntactic activation during language processing: A model based on neuropsychological and neurophysiological data. Brain and Language 50, 259-281. 
Friederici, A. D., 2002. Towards a neural basis for auditory sentence processing. Trends in Cognitive Science 6, 78-84.

Friederici, A. D., Hahne, A., Mecklinger, A., 1996. Temporal structure of syntactic parsing: Early and late event-related brain potential effects. Journal of Experimental Psychology: Learning, Memory and Cognition. 22, 1219-1248.

Frisch, S., Kotz, S. A., Von Cramon, D. Y., Friederici, A. D., 2003. Why the P600 is not just a P300: The role of the basal ganglia. Clinical neurophysiology $114,336-340$.

Gabrieli, J. D. E., Poldrack, R. A., Desmond, J. E., 1998. The role of the left pre-frontal cortex in language and memory. Proceedings of the National Academy of Sciences of the United States of America 95, 906-913.

Gunter, T. C., Stowe, L. A., Mulder, G., 1997. When syntax meets semantics. Psychophysiology 34, 660-676.

Haenschel, C., Vernon, D. J., Dwivedi, P., Gruzelier, J. H., Baldeweg, T., 2005. Event-related brain potential correlates of human auditory sensory memorytrace formation. The Journal of Neuroscience 25, 10494-10501.

Hagoort, P., Hald, L., Bastiaansen, M., Petersson, K. M., 2004. Integration of word meaning and world knowledge in language comprehension. Science 304, $438-441$.

Heike, A. E., 1981. A content-processing view of hesitation phenomena. Language and Speech 24, 147-160.

Holcomb, P. J., Neville, H. J., 1991. Natural speech processing: An analysis using event-related potentials. Psychobiology 19, 286-300.

Howell, P., Sackin, S., 2001. Function word repetitions emerge when speakers are operantly conditioned to reduce frequency of silent pauses. Journal of Psycholinguistic Research 30, 457-474. 
Jasper, H. H., 1958. Report to the committee on methods of clinical examination in electroencephalography. Appendix: The ten-twenty system of the international federation. Electroencephalography and Clinical Neurophysiology 10, $370-375$.

Kolk, H., Chwilla, D. J., van Herten, M., Oor, P. J. W., 2003. Structure and limited capacity in verbal working memory: A study with event-related potentials. Brain and Language 85, 1-36.

Kuperberg, G. R., 2007. Neural mechanisms of language comprehension: Challenges to syntax. Brain Research 1146, 23-49.

Kutas, M., Van Petten, C. K., Kluender, R., 2006. Psycholinguistics electrified II (1994-2005). In: Traxler, M., Gernsbacher, M. A. (Eds.), Handbook of Psycholinguistics, 2nd Edition. Elsevier Press, San Diego, CA, Ch. Psycholinguistics electrified II (1994-2005), pp. 83-143.

Ledoux, K., Traxler, M. J., Swaab, T. Y., 2007. Syntactic priming in comprehension: Evidence from event-related potentials. Psychological Science 18, $135-143$.

Maclay, H., Osgood, C. E., 1959. Hesitation phenomena in English speech. Word $15,19-44$.

McCarthy, G., Wood, C. C., 1985. Scalp distributions of event-related potentials: An ambiguity associated with analysis of variance models. Electroencephalography and Clinical Neurophysiology 62, 203-208.

Moreno, E. M., Federmeier, K. D., Kutas, M., 2002. Switching languages, switching palabras (words): An electrophysiological study of code switching. Brain and Language 80, 188-207.

Nagy, M. E., Rugg, M. D., 1989. Modulation of event-related potentials by word repetition: The effects of inter-item lag. Psychophysiology 26, 431-436. 
Okita, T., Jibu, T., 1998. Selective attention and N400 attenuation with spoken word repetition. Psychophysiology 35, 260-271.

Osterhout, L., Hagoort, P., 1999. A superficial resemblance does not necessarily mean you are part of the family: Counterarguments to Coulson, King and $\mathrm{Ku}-$ tas (1998) in the P600/SPS-P300 debate. Language and Cognitive Processes $14,1-14$.

Ranganath, C., Paller, K. A., 1999. Frontal brain activity during episodic and semantic retrieval: Insights from event-related potentials. Journal of Cognitive Neuroscience 11, 598-609.

Rhodes, S. M., Donaldson, D. I., 2008. Association and not semantic relationships elicit the N400 effect: Electrophysiological evidence from an explicit language comprehension task. Psychophysiology 44, 50-59.

Rugg, M. D., 1985. The effects of semantic priming and word repetition on event-related potentials. Psychophysiology 22, 642-647.

Rugg, M. D., Allan, K., Birch, C. S., 2000. Electrophysiological evidence for the modulation of retrieval orientation by depth of study processing. Journal of Cognitive Neuroscience 12, 664-678.

Salmon, N., Pratt, H., 2002. A comparison of sentence- and discourse-level semantic processing: An ERP study. Brain and Language 83, 367-383.

Senkfor, A. J., Van Petten, C. M., 1998. Who said what? An event-related potential investigation of source and item memory. Journal of Experimental Psychology: Learning, Memory, and Cognition 24, 1005-1025.

Shriberg, E. S., August 1995. Acoustic properties of disfluent repetitions. In: Proceedings of the International Congress of Phonetic Sciences. Vol. 4. Stockholm, Sweden, pp. 384-387.

Swaab, T. Y., Camblin, C. C., Gordon, P. C., 2004. Electrophysiological evidence for reversed lexical repetition effects in language processing. Journal Of Cognitive Neuroscience 16 (5), 715-726. 
Van Berkum, J. J. A., Brown, C. M., Hagoort, P., 1999. Early referential context effects in sentence processing: Evidence from event-related brain potentials. Journal of Memory and Language 41, 147-182.

Van Berkum, J. J. A., Brown, C. M., Hagoort, P., Zwitserlood, P., 2003. Eventrelated brain potentials reflect discourse-referential ambiguity in spoken language comprehension. Psychophysiology 40 (2), 235-248.

Van den Brink, D., Brown, C. M., Hagoort, P., 2001. Electrophysiological evidence for early contextual influences during spoken-word recognition: N200 versus N400 effects. Journal of Cognitive Neuroscience 13, 967-985.

Van Herten, M., Kolk, H. H. J., Chwilla, D. J., 2005. An ERP study of P600 effects elicited by semantic reversal anomalies. Cognitive Brain Research 22, $241-255$.

Van Herten, M., Kolk, H. H. J., Chwilla, D. J., 2006. When heuristics clash with parsing routines: ERP evidence for conflict monitoring in sentence perception. Journal of Cognitive Neuroscience 18 (2), 1-17.

Van Petten, C. M., Kutas, M., 1991. Influences of semantic and syntactic context on open and closed class words. Memory and Cognition 19, 95-112.

Van Petten, C. M., Kutas, M., Kluender, R., Mitchiner, M., McIsaac, H., 1991. Fractionating the word repetition effect with event-related potentials. Journal of Cognitive Neuroscience 3, 131-150.

Vissers, C. T. W. M., Chwilla, D. J., Kolk, H. H. J., 2006. Monitoring in language perception: The effect of misspellings of words in highly constrained sentences. Brain Research 1, 150-163. 